\title{
Problèmes d'ensablement des ports marocains sur la façade atlantique. Leur impact sédimentologique sur le littoral *
}

\author{
Mme Charrouf Latifa \\ Ingénieur des Ponts et Chaussées \\ Direction des Ports marocains
}

Les ports marocains sur la façade atlantique, et notamment ceux implantés au Sud du Cap Cantin où le transit littoral orienté Nord Sud varie de 250000 à $800000 \mathrm{~m}^{3} / \mathrm{an}$, connaissent des problèmes d'ensablement importants.

La plupart de ces ouvrages ont fait l'objet d'expérimentations sur modèles réduits physiques à fonds mobiles et d'un suivi des évolutions sédimentaires en nature.

Après avoir résumé les phénomènes naturels morphologiques, météorologiques, océanographiques et sédimentologiques le long de la côte, l'article donne les comparaisons entre les prévisions des modèles et les constatations a posteriori en nature et permet d'illustrer les différentes perturbations qu'un ouvrage portuaire peut apporter sur l'équilibre sédimentaire du littoral. On notera en particulier :

- l'influence de la profondeur d'implantation d'un ouvrage maritime ;

- la formation de flèches sédimentaires et de tombolos;

- l'accumulation du transit littoral ;

- l'influence des barres;

- le transport par le courant d'expansion latérale ;

- et d'une façon générale, l'impact sédimentologique de l'ouvrage sur le littoral.
Cet article permet également d'apprécier l'importance des études sur modèle réduit physique et leur validité, à condition qu'elles soient basées sur des mesures préliminaires très poussées et complètes, tant sur l'océanographie que sur la sédimentologie.

\section{Répartition morphologique du littoral}

Le long du littoral atlantique marocain (2 $500 \mathrm{~km} \mathrm{de}$ Tanger à Lagwira) où ont été réalisés 22 ports de 1900 à 1988, différents types de côtes se rencontrent, allant des plages avec ou sans dunes, telles que celles entre Cap Spartel (Asilah) et le Sud d'Agadir, à des côtes rocheuses avec ou sans criques, pour atteindre des côtes à falaises de plusieurs dizaines de mètres de hauteur (Safi, Cap Blanc, Sidi Ifni, Cap Juby, etc... fig. l).

(*) Extrait de la thèse de doctorat soutenue le 6 juillet 1989 à l'Université Paris-Sud, centre d'Orsay.

\section{Problems of silting in Moroccan ports on the Atlantic side}

The sedimentological impact on the coast

Moroccan ports on the Atlantic side and especially those to the South of Cap Cantin where the coast passage orientated North South varies from 250000 to $800000 \mathrm{~m}^{3} /$ year are having especially difficult silting and sand problems. Most of these works have been experimented on reduced physical models with mobile bottoms and have also monitored the sediment trends in the natural setting. After summing up the natural morphological, meteorological, oceanographic and sedimentological phenomena along the coast, this article compares forecasts from models with observations after the event in the natural settings and thus helps to illustrate the different perturbations that port works may cause to the sediment equilibrium of the coast. 


\section{Météorologie et océanographie}

La côte atlantique marocaine est soumise à des conditions naturelles qui sont résumées ci-après.

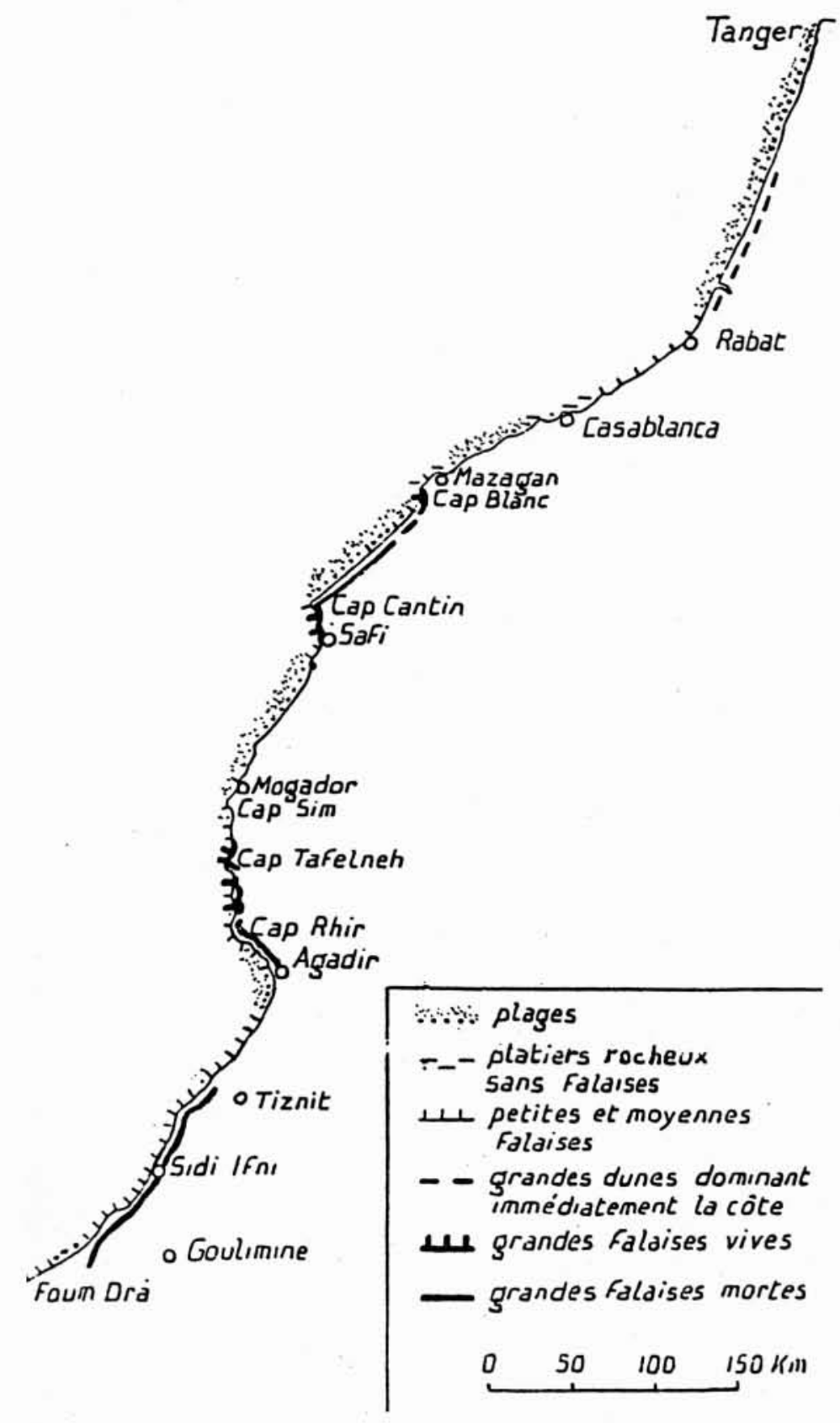

1. Divisions morphologiques de la côte atlantique marocaine de Tanger au Cap Draa

\subsection{Vents}

La répartition des vitesses des vents au cours de l'année fait apparaître une fréquence élevée des vents supérieurs à $8 \mathrm{~m} / \mathrm{s}$ dans les provinces au Sud d'Agadir et à Tanger.

$\mathrm{La}$ fréquence des vents faibles (inférieurs à $2 \mathrm{~m} / \mathrm{s}$ ) et modérés (entre 2 et $10 \mathrm{~m} / \mathrm{s}$ ) est élevée dans le secteur central Casablanca-Safi.

\subsection{Courants}

Les courants généraux et les courants de marée en dehors des embouchures des Oueds peuvent être négligés. Les courants susceptibles d'agir sur le transit sédimentaire le long de la côte atlantique marocaine naissent principalement de l'action des houles.

Dans la région centrale Larache-Jorf Lasfar, le régime des courants est essentiellement dominé par les vents. Les vitesses, de l'ordre de 2,5 à $3 \%$ de la vitesse des vents, peuvent atteindre exceptionnellement $(0,50 \mathrm{~m} / \mathrm{s})$ dans la région de Mohammedia.

Au Sud du Cap Cantin, les courants de houles sont responsables des transports des sables. Les valeurs peuvent atteindre $1,5 \mathrm{~m} / \mathrm{s}$ dans la zone de déferlement et portent au Sud.

Des rip - currents atteignant $2 \mathrm{~m} / \mathrm{s}$ ont pu être mesurés à Sidi Ifni par fortes houles. Au Sud du Cap Juby, cette zone est soumise à un courant constant en direction de $0,25 \mathrm{~m} / \mathrm{s}$. Il est possible par vent du Nord que ce courant soit l'agent de transport de matériaux remis en suspension sous l'action de la houle.

\subsection{Marée}

La marée est du type semi diurne régulier, les hauteurs des plus fortes marées sur la côte atlantique du Maroc dépassent légèrement $4 \mathrm{~m}$, les plus faibles valent environ $1 \mathrm{~m}$.

Des phénomènes météorologiques et océanographiques peuvent modifier sensiblement les prévisions de l'annuaire des marées et conduire à des surélévations qui peuvent atteindre exceptionnellement 0,5 à $1 \mathrm{~m}$.

\subsection{Houles}

Les houles au large sur la côte Cap Spartel-Agadir proviennent du secteur SSW à NNW avec une prédominance du secteur W-NW.

Dans la région Cap Spartel Jorf-Lasfar, la direction sur les sites est pratiquement perpendiculaire à la côte. Ceci n'est plus le cas au Sud du Cap Cantin où l'on rencontre par exemple à Safi une prédominance de WqNW à NW $(58 \%)$ et W à WSW (50\%) à Agadir.

Au Sud d'Agadir la prédominance au large est au secteur WNW à N jusqu'au Cap Juby, et au NNW à NW au Sud du Cap précité.

Les caractéristiques des houles mesurées ou observées sur les ports marocains atlantiques sont données dans les tableaux I et II. 
Tableau I

Fréquences cumulées des amplitudes de la houle

\begin{tabular}{|c|c|c|c|c|c|c|c|}
\hline $\begin{array}{l}\text { Amplitude }(\mathrm{m}) \\
\text { Stations }\end{array}$ & $H \geqslant 0$ & $H \geqslant 0,5$ & $H \geqslant 1,5$ & $H \geqslant 3$ & $H \geqslant 5$ & $H \geqslant 7$ & Observations \\
\hline Mehdia & 100 & 96,4 & 26,3 & 6,8 & 0,60 & 0,05 & \multirow{6}{*}{$\begin{array}{l}\text { - Observations de SIMONET et TANGUY } \\
\text { (1928-1952) } \\
\text { - Les amplitudes observées peuvent } \\
\text { être considérées par mesure de sécu- } \\
\text { rité comme des amplitudes moyennes } \\
\text { maximums de la houle libre déferlante } \\
\text { ou non }\end{array}$} \\
\hline Rabat & 100 & 92,9 & 29,3 & 6,7 & 0,44 & 0,11 & \\
\hline Casablanca & 100 & 93,6 & 24,2 & 3,7 & 0,41 & 0,08 & \\
\hline Safi & 100 & 96,4 & 25,2 & 3,6 & 0,41 & 0,08 & \\
\hline Essaouira & 100 & 96,6 & 47,9 & 5,5 & 0,60 & 0,16 & \\
\hline Agadir & 100 & 97,2 & 22,1 & 3,8 & 0,22 & 0,03 & \\
\hline Tanger (Hs) & 100 & 78,9 & $30,88^{*}$ & 0,23 & $0,15^{\star}$ & - & $\begin{array}{l}\text { Enregistrements récents pour les } \\
\text { études de la liaison fixe du Détroit de } \\
\text { Gibraltar, sept. } 87-88\end{array}$ \\
\hline Larache $(\mathrm{Hs})$ & 100 & 96,4 & 26,3 & 6,8 & 0,6 & - & Etude L.C.H.F. 1977 \\
\hline Mohammédia (Hs) & 100 & 99,91 & 53,09 & 7,51 & 0,60 & 0,09 & $\begin{array}{l}\text { Mesures G.I.S. lors des travaux d'ex- } \\
\text { tension de Mohammédia }\end{array}$ \\
\hline Jorf Lasfar (Hs) & - & 100 & 52,59 & 11,11 & 1,75 & 0,24 & $\begin{array}{l}\text { Enregistrements effectués à Jorf Lasfar, } \\
\text { mai 1978-mars } 1982\end{array}$ \\
\hline Sidi Ifni (Hs) & 100 & 99,19 & 48,3 & 6,59 & 0,55 & - & Etude L.C.H.F. 1976 \\
\hline Tarfaya-Laayoune & 100 & 92,4 & $21,8^{\star \star}$ & 4,5 & $0,9^{* *}$ & $0,05^{\star *}$ & $\begin{array}{l}\text { Observations de navires sur une } \\
\text { période de } 20 \text { ans } 1954-74\end{array}$ \\
\hline \multicolumn{8}{|c|}{$\begin{array}{l}\text { Nota: } \\
\text { Valeurs donnant les fréquences cumulées des houles respectivement }>\text { à } 1,0 \text { et } 4,0 \mathrm{~m} \text {. } \\
\text { * Valeurs donnant les fréquences cumulées des houles respectivement }>\text { à } 2,0-4,0 \text { et } 6,0 \mathrm{~m} \text {. }\end{array}$} \\
\hline
\end{tabular}

Tableau II

Répartition des périodes des houles (pourcentages non cumulés)

\begin{tabular}{|c|c|c|}
\hline \multirow[b]{2}{*}{$\begin{array}{l}\text { Périodes } T \\
\text { en (s) }\end{array}$} & \multicolumn{2}{|c|}{ Secteurs } \\
\hline & $\begin{array}{c}\text { Mehdia, Casa, } \\
\text { Jorf Lasfar, } \\
\text { Safi, Agadir }\end{array}$ & Au Sud Cap-Juby \\
\hline$<9 \mathrm{~s}$ & 20 à $31 \%$ & 64 à $66 \%$ \\
\hline $9-13 s$ & 55 à $65 \%$ & 29 à $33 \%$ \\
\hline$>13 \mathrm{~s}$ & 8 à $17 \%$ & 3 à $4 \%$ \\
\hline
\end{tabular}




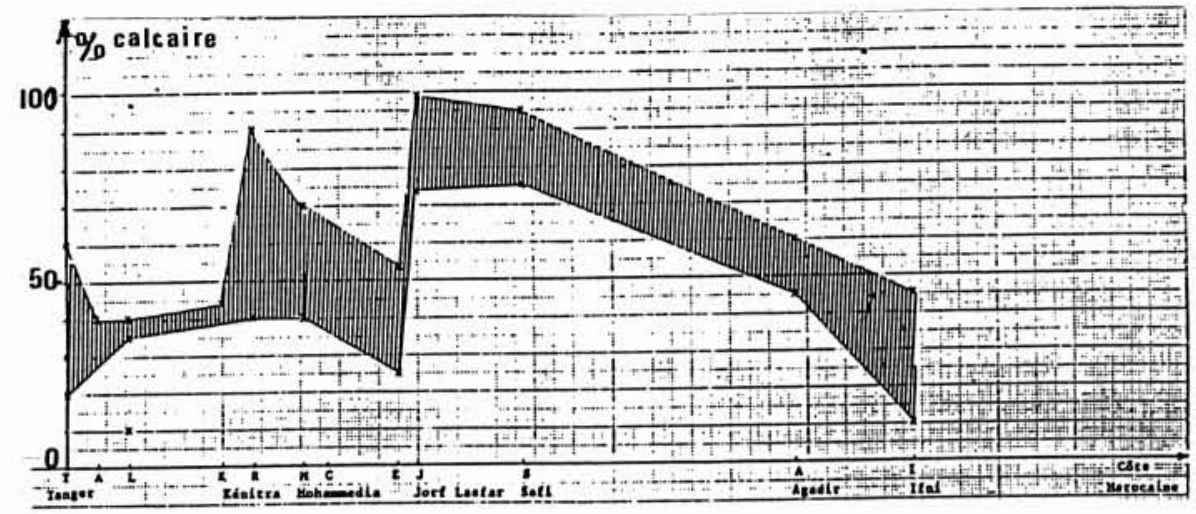

2. Variation de la teneur en calcaire des sédiments

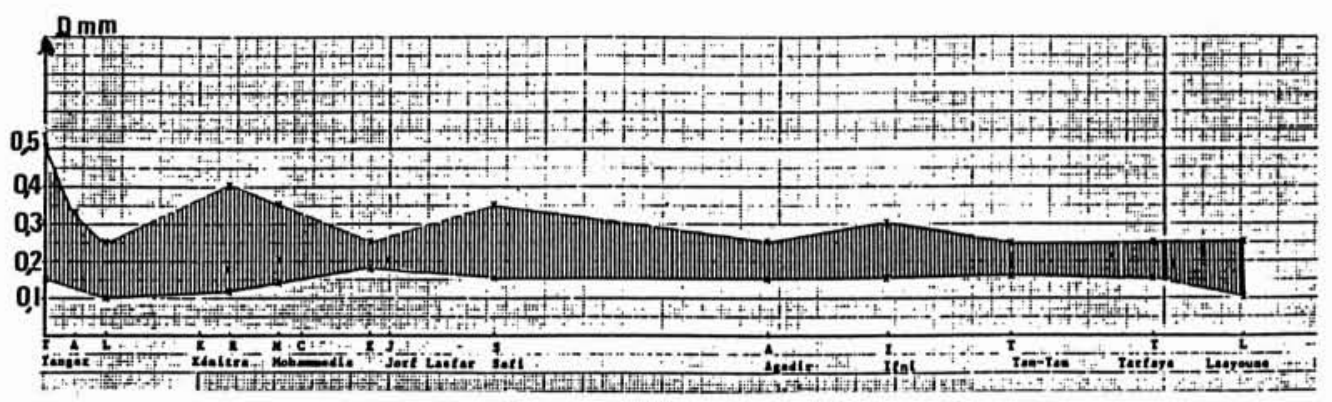

3. Variation de la granulométrie des sédiments

\section{Sédimentologie et mouvements sédimentaires}

\subsection{Caractéristiques des sédiments}

Les sédiments que l'on trouve sur la côte atlantique marocaine diffèrent en nature d'une région à une autre. La variation de la teneur en calcaire fait apparaître des sables silteux au Nord, des sables entièrement coquilliers dans le secteur Rabat-Safi (70 à $99 \%$ ), pour revenir à des sédiments riches en éléments détritiques au Sud (fig. 2).

Le fuseau granulométrique le long de cette côte montre que les sédiments sont généralement fins. La vitesse de chute en eau calme varie de 1 à $3 \mathrm{~cm} / \mathrm{s}$ (fig. 3 ).

Ces matériaux sont remaniés par les courants dès que la contrainte tangentielle exercée par l'eau sur les fonds dépasse $0,2 \mathrm{~N} / \mathrm{m}^{2}$ en moyenne.

\subsection{Estimation des mouvements sédimentaires}

Transports fluviaux: Plus de 20 Oueds débouchent sur la côte atlantique marocaine, le plus important étant celui du Sébou. Les apports solides à la mer sont estimés à environ 33 millions de tonnes annuellement. Les sables et galets ne doivent guère représenter plus de $10 \%$ de l'ensemble des apports, soit 3 millions de tonnes par an. La majeure partie de ces apports se fait lors des crues et, du fait des fortes vitesses de l'écoulement en mer de ces crues, il est probable qu'un certain pourcentage des apports solides se perd vers le large (la totalité pour les vases, une bonne part pour les sables et peu pour les galets).

Transports éoliens : Ces transports, en général d'ouest en est, ne sont significatifs que dans la région de Tanger Asilah, et au Sud d'Agadir avec des intensités très élevées au Sud du Cap Juby.

\begin{tabular}{|c|c|c|c|}
\hline Sites & $\begin{array}{c}\text { Baie } \\
\text { de Tanger }\end{array}$ & $\begin{array}{c}\text { Oueds } \\
\text { Souss- } \\
\text { Massa }\end{array}$ & $\begin{array}{c}\text { Au Sud } \\
\text { du Cap } \\
\text { Juby }\end{array}$ \\
\hline $\begin{array}{l}\text { Taux } \mathrm{m}^{3} \text { /an par } \\
\text { mètre linéaire }\end{array}$ & 5 & 30 à 50 & 160 \\
\hline
\end{tabular}

Mouvements sédimentaires sous l'action des houles : Ces mouvements peuvent être décomposés en deux types:

- perpendiculairement à la côte, dans le profil, ceux-ci sont faibles au-delà des fonds de $-15 \mathrm{~m}$ hydro ;

— parallèlement à la côte : le transit littoral dominant est orienté Nord Sud. Le transit Sud Nord est en général faible (tabl. III).

Il y a lieu de préciser que les apports sédimentaires s'effectuent presque uniquement en quelques jours de tempêtes. En effet seules les houles de tempêtes (amplitudes 
dépassant $2 \mathrm{~m}$ avant déferlement) créent dans la zone de déferlement de violents courants susceptibles de transporter d'importantes masses de sables. Les différentes observations et études ont montré que :

- à Agadir, $25 \%$ du transit littoral se répartissent dans la période d'été (mai à septembre) et $75 \%$ en hiver (octobre à avril), les mois de décembre et janvier représentant à eux seuls $45 \%$ des apports annuels. Une houle forte à moyenne $(\mathrm{H}=2$ à $2,5 \mathrm{~m})$ peut transporter en 15 jours $150000 \mathrm{~m}^{3}$. En 7 heures, une houle de $3 \mathrm{~m}$ d'amplitude et $12 \mathrm{sec}$ de période a transporté $35000 \mathrm{~m}^{3}$ les 29 et 30 novembre 1947 ;

- à Safi, la SEHOM et le Laboratoire Central d'Hydraulique de France (L.C.H.F.) avaient constaté que les tempêtes des 2-3 janvier 1949 et février 1966 ont transporté respectivement 80000 et $110000 \mathrm{~m}^{3}$.

De même, un suivi régulier du piège à sable à Safi en 1986 a montré que $73 \%$ du transit total $\left(180000 \mathrm{~m}^{3}\right)$ se

\section{Tableau III}

\begin{tabular}{|c|c|c|}
\hline Sites & $\begin{array}{c}\text { Valeur } \\
\text { du transit } \\
\text { littoral résultant } \\
\text { Nord-Sud en } \mathrm{m}^{3} \\
\text { par an }\end{array}$ & Origine \\
\hline Baie de Tanger & 35000 & $\begin{array}{l}\text { Apports organi- } \\
\text { ques fluviaux } \\
\text { érosion marine }\end{array}$ \\
\hline $\begin{array}{l}\text { Cap spartel } \\
\text { Cap Blanc }\end{array}$ & $\begin{array}{l}\text { Quelques dizai- } \\
\text { nes de milliers }\end{array}$ & $\begin{array}{l}\text { Apports marins } \\
\text { essentiellement }\end{array}$ \\
\hline Safi & 250000 & $\begin{array}{l}\text { Erosion par mer } \\
\text { des dunes et } \\
\text { plages quater- } \\
\text { naires Oualidia- } \\
\text { Cap Cantin }\end{array}$ \\
\hline Agadir & 450 à 500000 & $\begin{array}{l}\text { Erosion des } \\
\text { falaises (Cap } \\
\text { Ghir-Arhesdis) } \\
\text { et apports du } \\
\text { large, et des } \\
\text { oueds }\end{array}$ \\
\hline Sidi Ifni & 400000 & $\begin{array}{l}\text { Erosion des } \\
\text { falaises Nord, } \\
\text { apports des } \\
\text { oueds, apports } \\
\text { marins }\end{array}$ \\
\hline Tan-Tan & 450000 & $\begin{array}{l}\text { Plage Blanche } \\
\text { et apports des } \\
\text { oueds au Nord }\end{array}$ \\
\hline Tarfaya & 400000 & $\begin{array}{l}\text { Dunes et } \\
\text { apports marins }\end{array}$ \\
\hline Laayoune & 500 à 800000 & $\begin{array}{l}\text { Plages et dunes } \\
\text { au Nord }\end{array}$ \\
\hline
\end{tabular}

sont déposés au cours de tempêtes de durée cumulée égale à 10 jours.

Les houles moyennes et de beau temps ont pour effet de remodeler les petits fonds et l'estran, mais ne transportent pas parallèlement au rivage de grosses quantités de sable.

\section{Les différents types d'aménagements portuaires sur la côte atlantique marocaine}

Les aménagements portuaires réalisés sur la côte atlantique marocaine sont très diversifiés et l'on retrouve toutes les grandes catégories d'ouvrages et les différents phénomènes et comportements sédimentologiques (fig. 4):

- ports en eau profonde sur une côte à faible transit littoral ;

- ports implantés par grandes profondeurs sur une côte où le transit littoral est important et qui interceptent la majeure partie de ce transit;

— ports implantés par profondeurs moyennes (près de la barre);

— ports implantés près du rivage à l'intérieur de la fosse de lévigation ;

- ports qui modifient fortement l'expansion latérale de la houle ;

— enfin, les aménagements portuaires sur les estuaires.

- Ports en eau profonde sur une cote a falble transit littoral

o. Ports Implantés par grandes profondeurs sur une cote où le transit Littaral est 1rportant.

5. Ports implantés par profondeurs moyennea (près de la barre).

a. Ports inplantés près du rivage a l'intérieur de la fosse de lévigation. 7. Anénagenents portualies sur les estuaires.

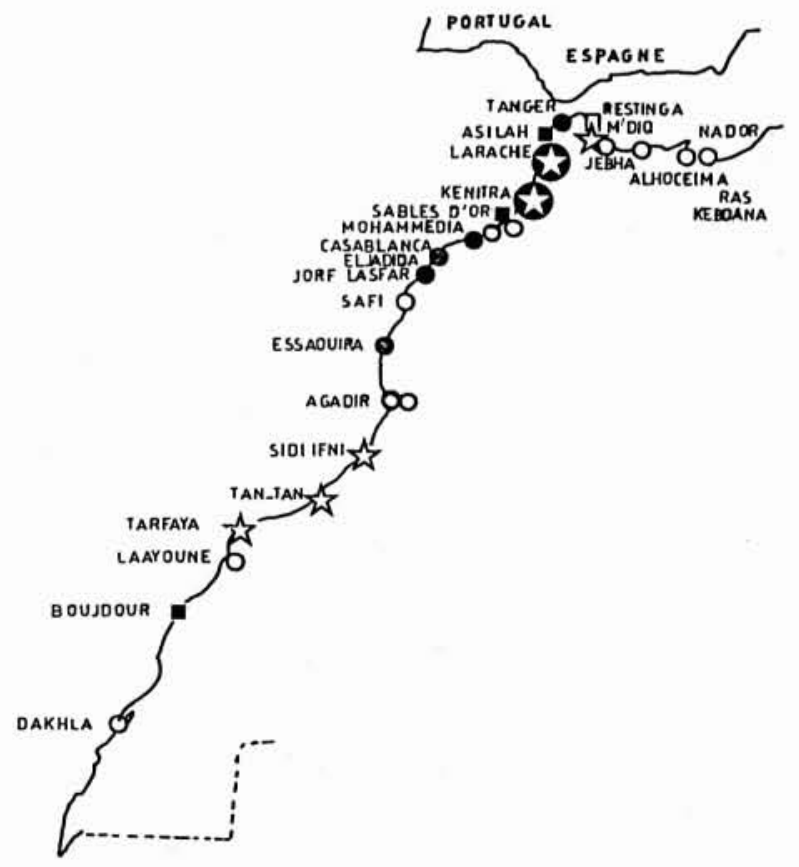

4. 


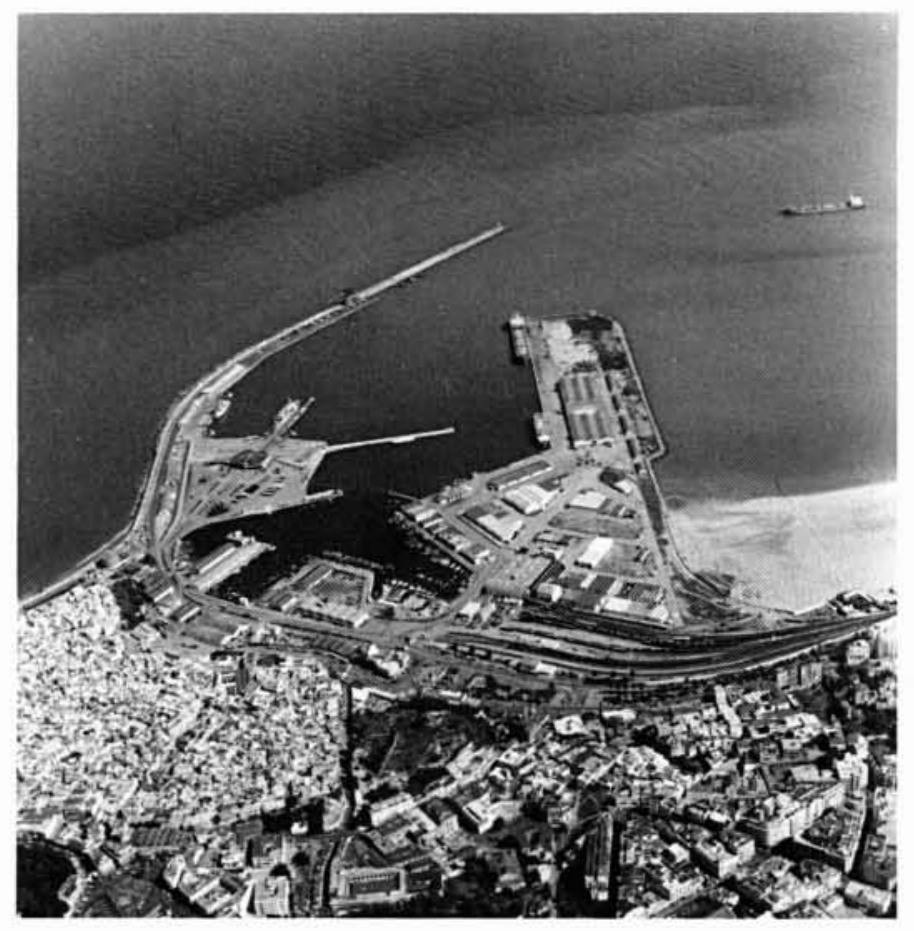

5. Port de Tanger

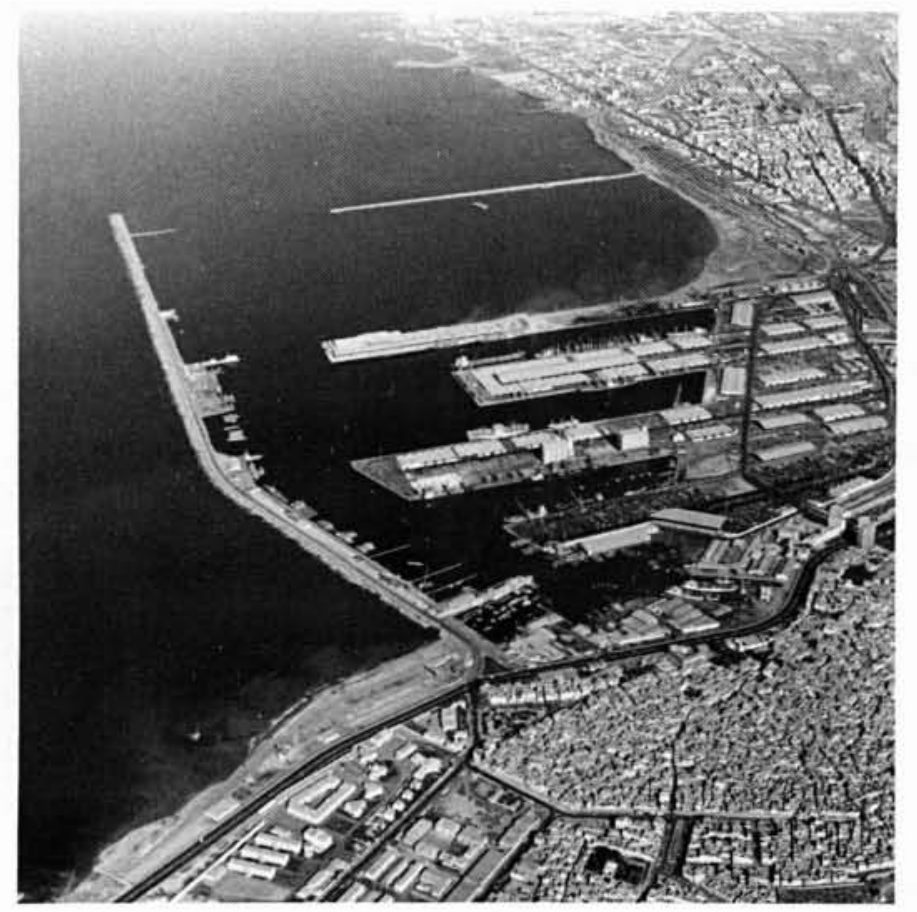

6. Port de Casablanca

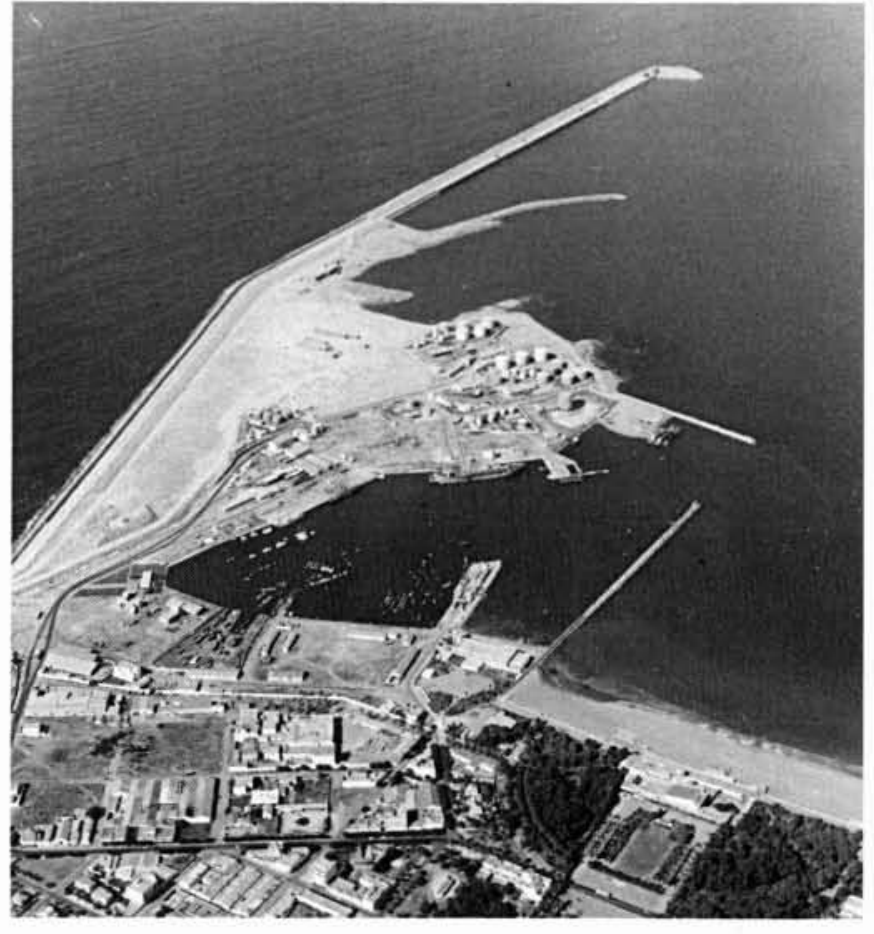

7. Port de Mohammédia

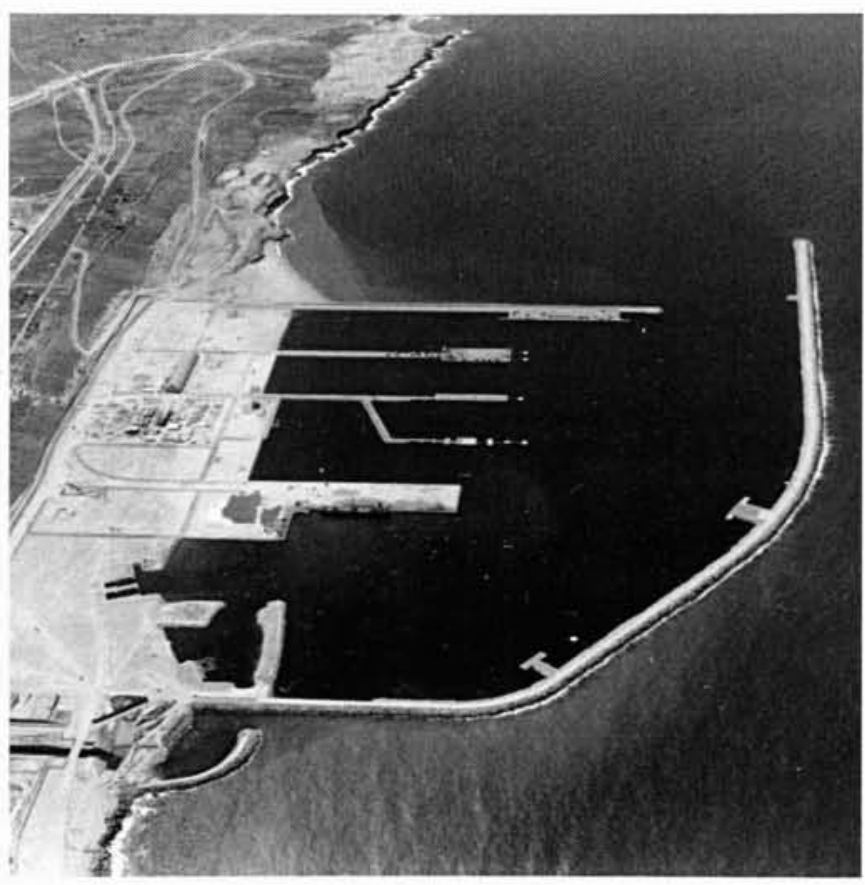

8. Port de Jorf Laslar 


\section{Ports implantés par grandes profondeurs sur une côte à faible transit littoral}

Quatre ports forment cette classe: Tanger (fig. 5), Casablanca (fig. 6), Port pétrolier de Mohammédia (fig. 7) et Jorf Lasfar (fig. 8).

Les caractéristiques sont données dans le tableau $\mathrm{IV}$.

L'ensablement des quatre ports précités reste très faible, inférieur à $0,30 \mathrm{~m}$ par an.

Les ouvrages de protection des ports de Tanger, Mohammédia et Jorf, Lasfar coupant la propagation de la houle induisent dans la zone abritée un courant d'expan- sion latérale orienté du secteur de forte agitation vers le secteur de faible agitation. Il en résulte un engraissement des plages adossées aux jetées transversales atteignant :

- $20000 \mathrm{~m}^{3} /$ an à Jorf Lasfar (fig. 9);

- $60000 \mathrm{~m}^{3} / \mathrm{an}$ à Tanger (dont $30 \%$ de transports éoliens). Ce volume devrait diminuer par suite de la réalisation de la protection de la plage Est en 1985 et des mesures prises contre l'érosion éolienne (fig. 10);

— plus de $50000 \mathrm{~m}^{3} /$ an à Mohammédia.

L'impact sédimentologique des ports de Casablanca et Jorf Lasfar sur le littoral avoisinant reste faible ; celui des ports de Tanger et Mohammédia, loin d'être négligeable, s'est traduit par des érosions importantes des plages Est.

Tableau IV

\begin{tabular}{|l|c|c|c|c|}
\hline \multicolumn{1}{|c|}{ Ports } & $\begin{array}{c}\text { Surface du } \\
\text { plan d'eau }\end{array}$ & $\begin{array}{c}\text { Longueur et profondeur } \\
\text { de la jetée principale }\end{array}$ & $\begin{array}{c}\text { Longueur et profondeur } \\
\text { de la jetée transversale }\end{array}$ & Ouverture du port \\
\hline Tanger & $68 \mathrm{ha}$ & $1320 \mathrm{~m}(-14 \mathrm{~m})$ & $1000 \mathrm{~m}(-12 \mathrm{~m})$ & $\mathrm{E}$ \\
\hline Casablanca & $200 \mathrm{ha}$ & $3180 \mathrm{~m}(-18 \mathrm{~m})$ & $1500 \mathrm{~m}(-13 \mathrm{~m})$ & NE \\
\hline Mohammédia & $180 \mathrm{ha}$ & $2800 \mathrm{~m}(-27 \mathrm{~m})$ & $500 \mathrm{~m}(-15 \mathrm{~m})$ & NE \\
\hline Jorf Lasfa & $200 \mathrm{ha}$ & $3100 \mathrm{~m}(-17 \mathrm{~m})$ & $1250 \mathrm{~m}(-13 \mathrm{~m})$ & $\mathrm{SW}$ \\
\hline
\end{tabular}

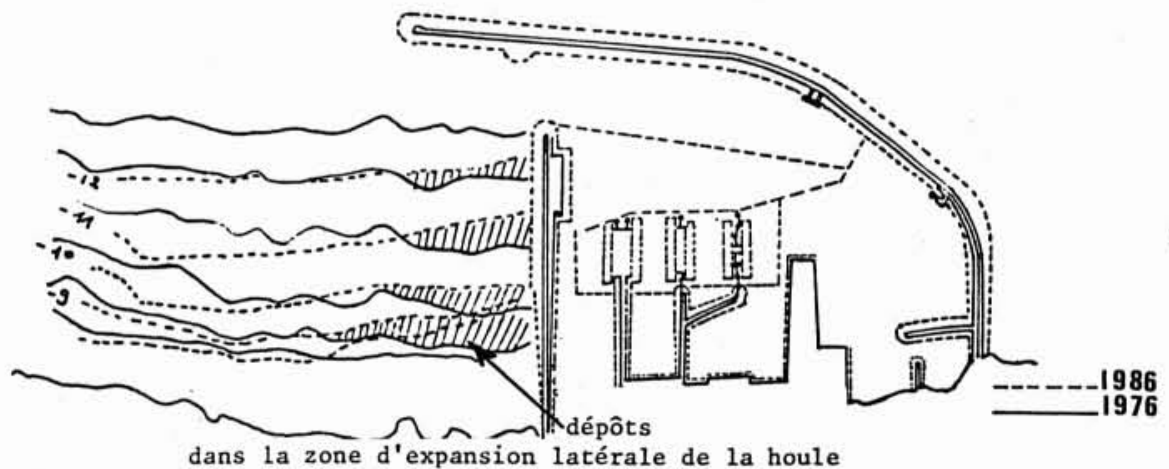

9. Evolution des fonds au port de Jorf Lasfar entre 1976 et 1986

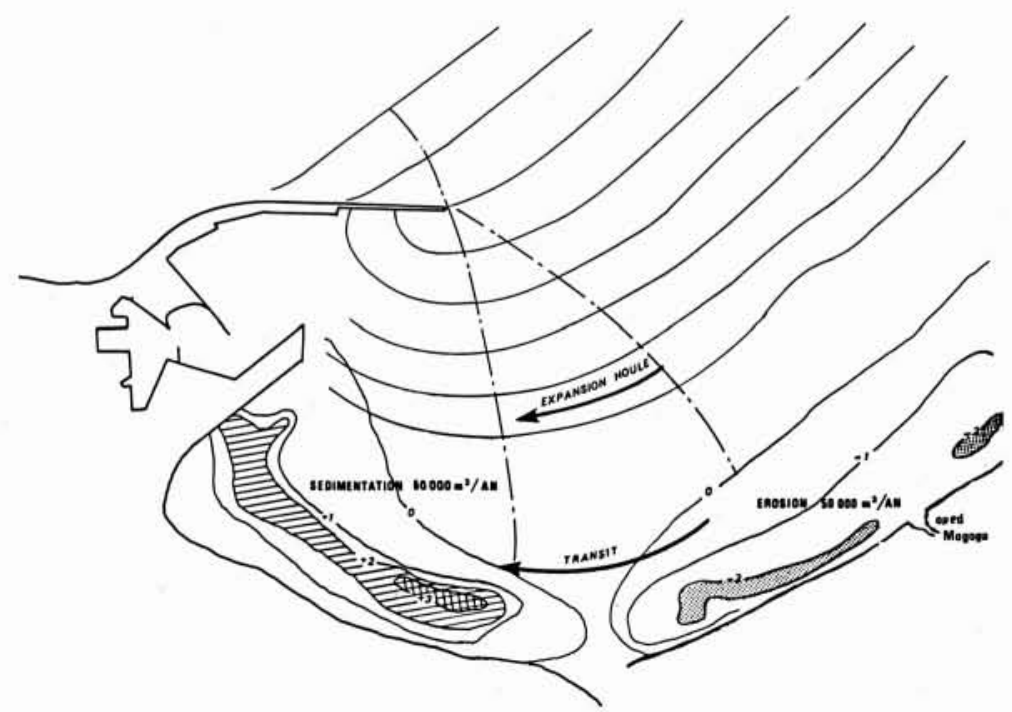

10. Apports sédmentaires par le courant d'expansion latérale de la houle à Tanger 


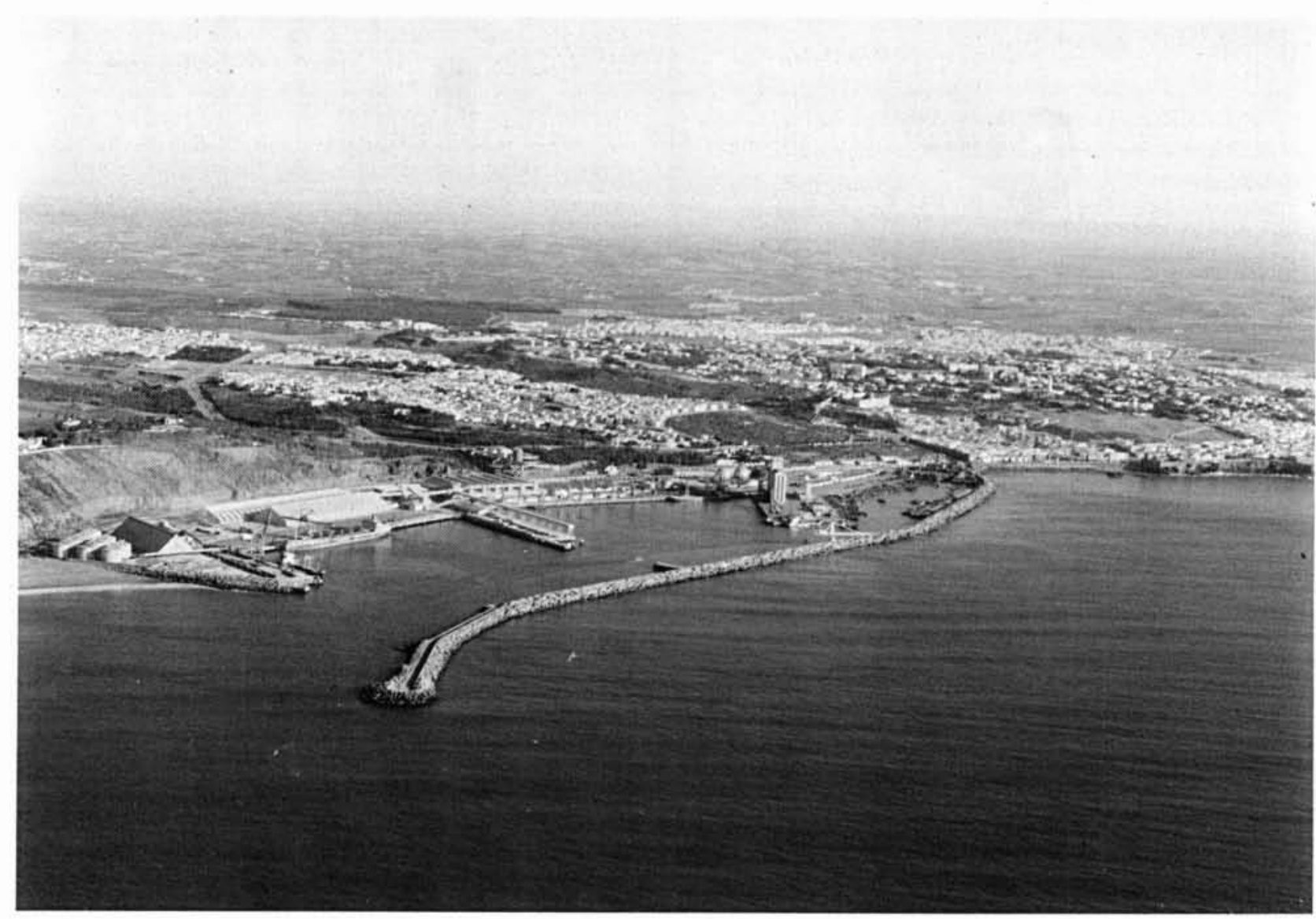

11. Port actuel de Safi

\section{Ports implantés par grandes profondeurs sur une côte où le transit littoral est important}

\subsection{Port ouvert au transit littoral}

Le Port de Safi est protégé par une jetée principale de $2 \mathrm{~km}$ environ qui longe l'isobathe $-13 \mathrm{~m}$. Côté Nord. Il est limité par une jetée transversale de $500 \mathrm{~m}$. Il est ouvert au NW (fig. 11).

Au fur et à mesure de leur construction, les ouvrages du Port de Safi ont provoqué une accumulation de sable sur leur face Nord en arrêtant la plus grande partie du transit sédimentaire. Le phénomène d'ensablement se reproduisait toujours de la même manière ; les sédiments se déposaient dans l'angle formé par la jetée transversale et la côte en donnant à la plage une forme en croissant dont l'une des branches avait tendance à s'allonger le long de cette jetée contre laquelle les atterrissements étaient les plus importants. L'engraissement se faisait sentir jusqu'à des profondeurs importantes de -8 à $-12 \mathrm{~m}$ (fig. 12).

Les possibilités d'emmagasinement des sables le long de la jetée transversale dépendent de l'implantation de cette dernière par rapport à la configuration du trait de côte et de l'orientation générale des houles dominantes (W à WqNW sur le site).

Après la saturation, les sédiments peuvent atteindre la passe et la colmater, une plage sous marine curviligne ayant tendance à se dessiner au fond de cette enclave artificielle. Il a été constaté que si l'on ne draguait pas continuellement le piège à sable, les sédiments s'avanceraient le long de la passe d'entrée et dessineraient jusqu'à la jetée, qu'ils finiraient par rejoindre, un cordon circulaire.

Des dragages de 200 à $250000 \mathrm{~m}^{3} /$ an avec creusement de fosse de garde ont été nécessaires pour maintenir les accès au port.

Les solutions pour réduire les risques d'ensablement de la passe d'entrée et des accès au port sont nombreuses :

- arrêter le transit littoral par des ouvrages réalisés à l'amont du port à 1 ou 1,5 km (épis ou brise-lames) ou par une nouvelle jetée transversale implantée au saillant carrière (fig. 13);

- réaliser une fosse de garde dans la partie extrême de la jetée secondaire. Cette solution a l'avantage d'éviter un ensablement brutal de la passe d'entrée du port au cours des tempêtes. La capacité de la fosse peut varier de 1,5 à 2 fois le volume annuel du transit. 


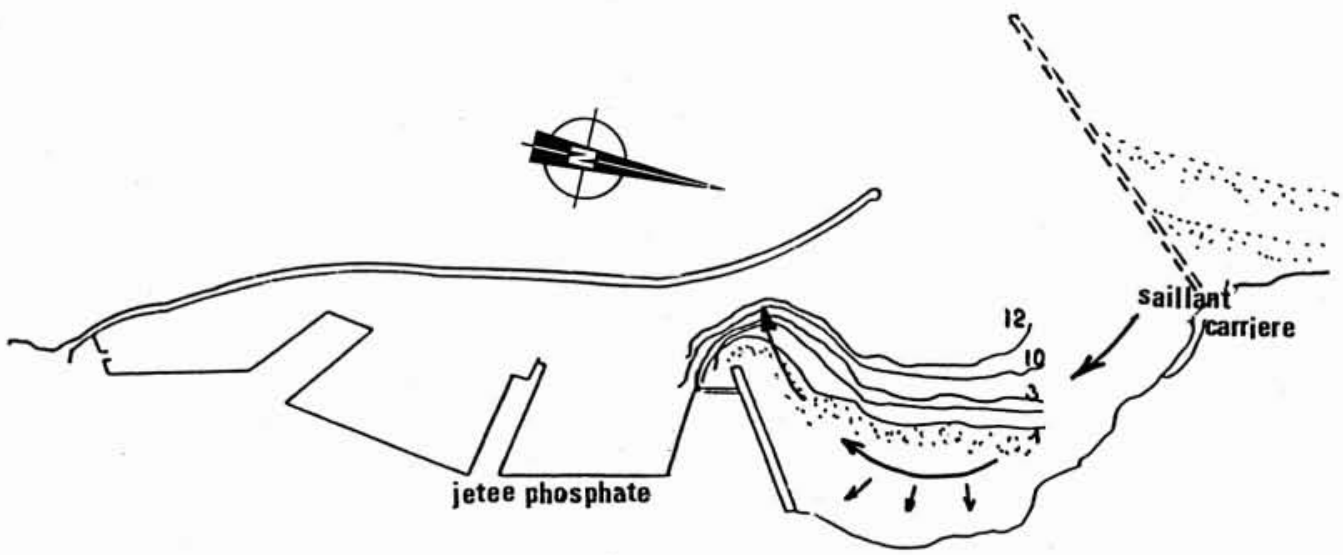

12. Accumulation des sables sur la plage au nord du port de Safi et risque de colmatage de la passe d'entrée

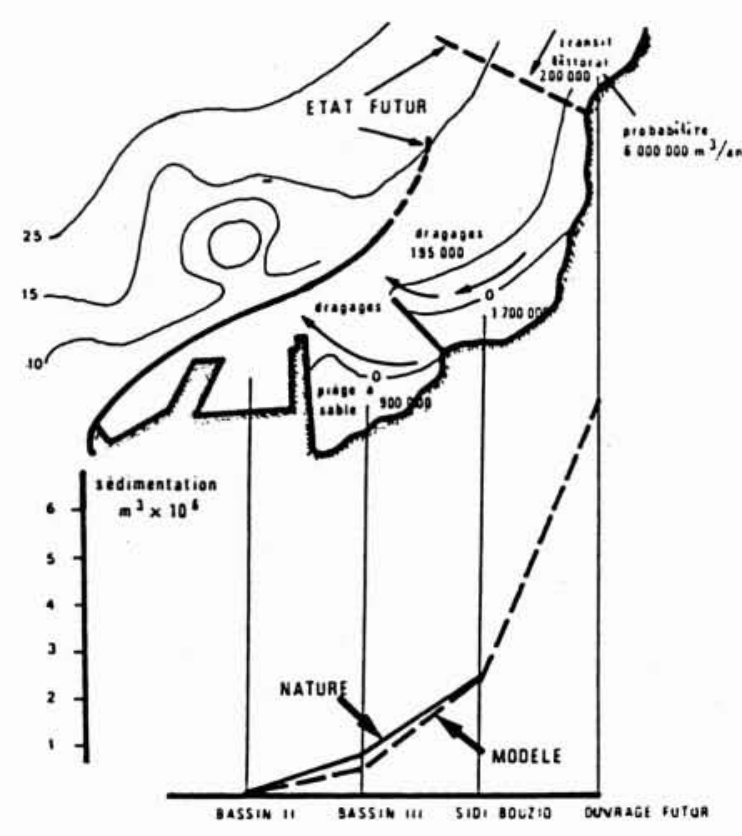

13. Arrêt des sédiments par une jetée réalisée au nord du port de Safi

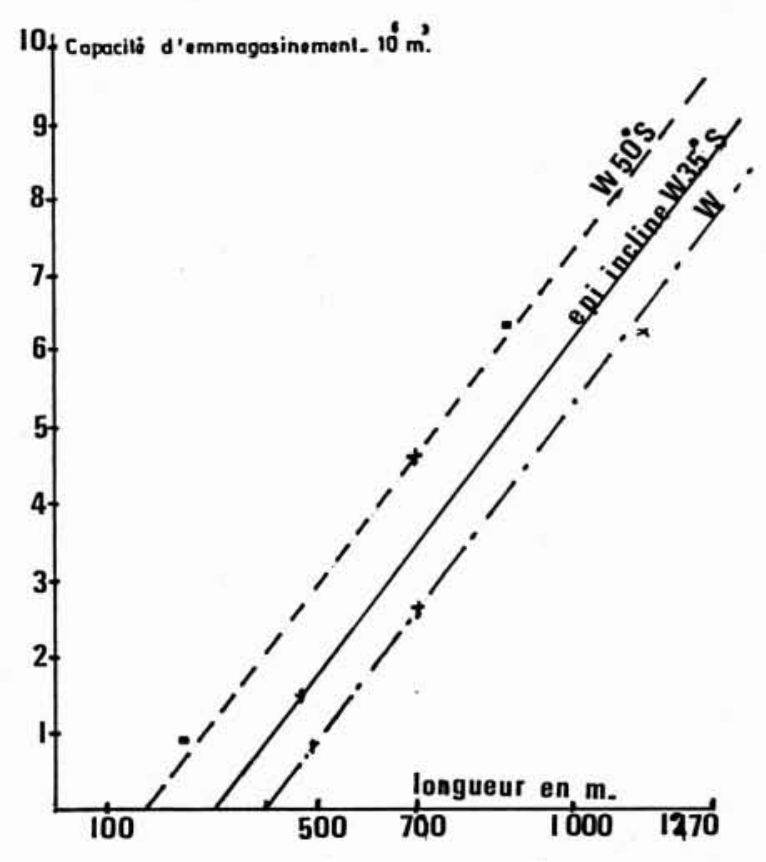



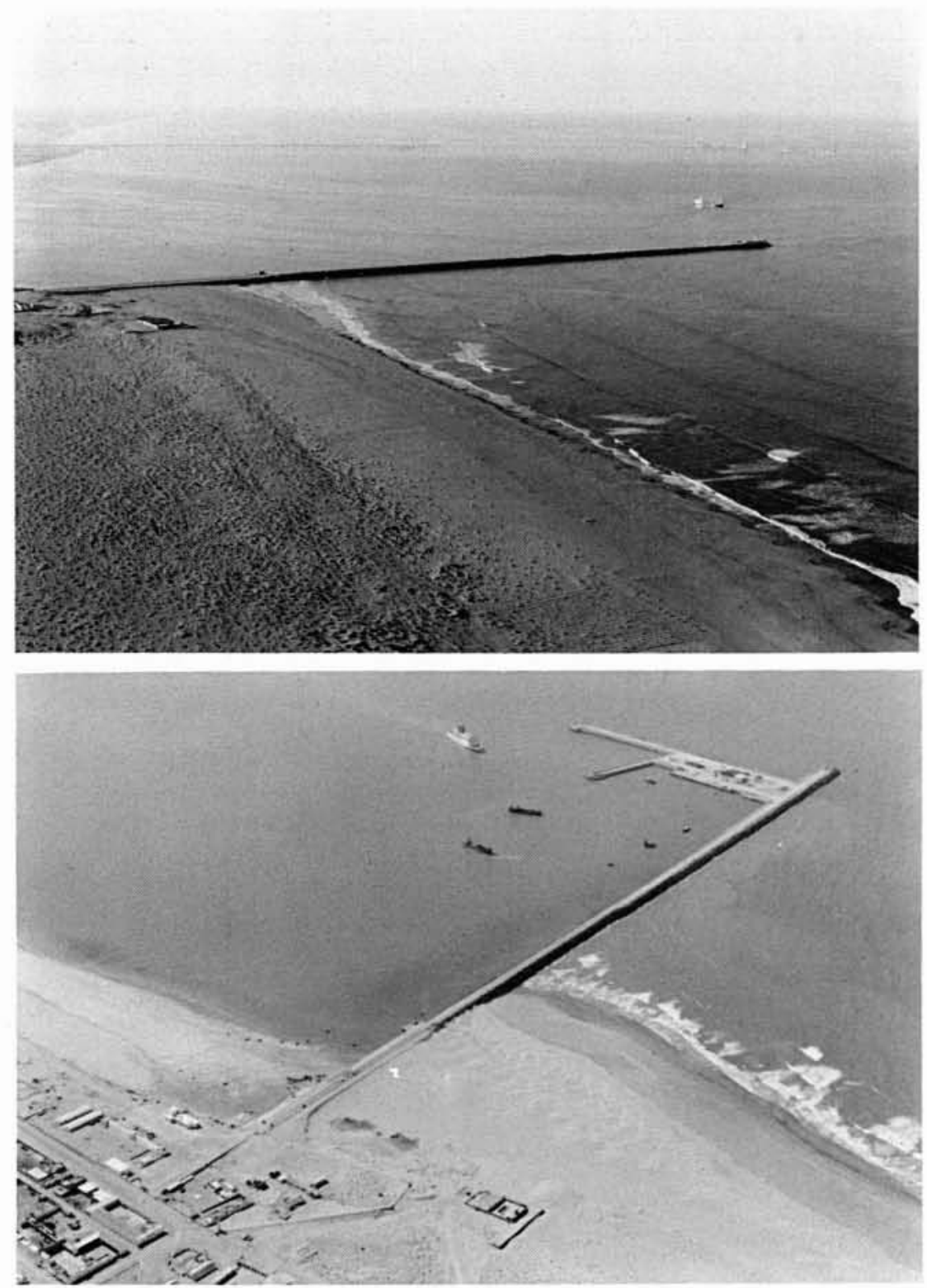

14. Port de Laayoune en cours de construction

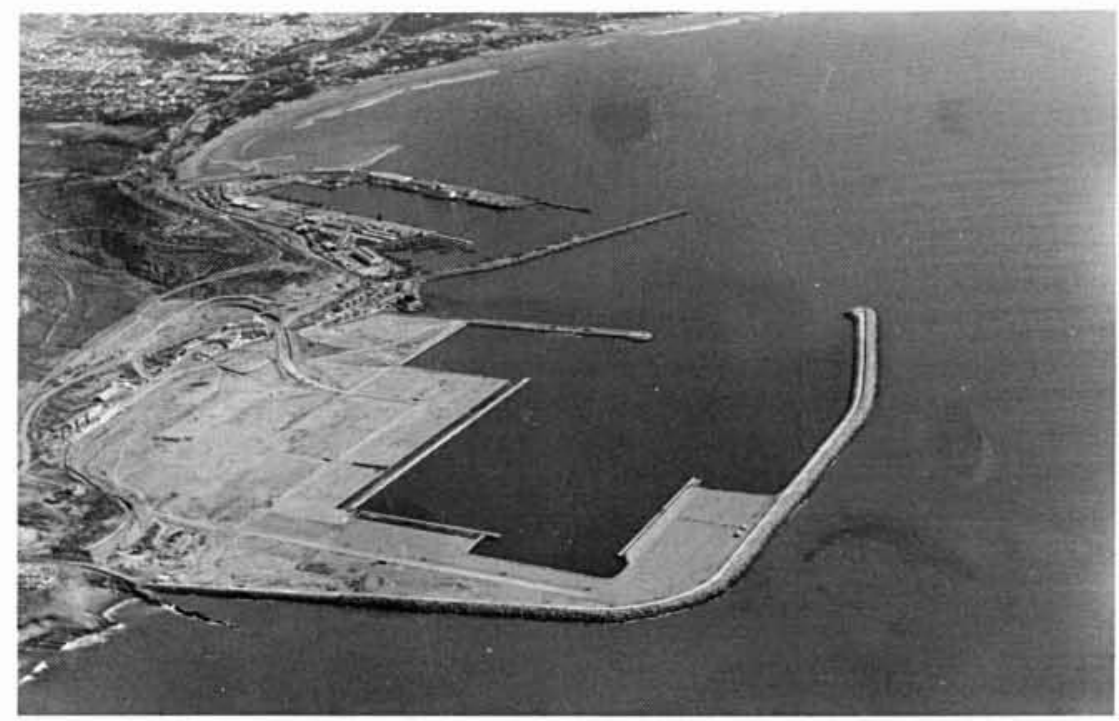

15. Nouveau complexe portuaire d'Agadir-Anza 
Tableau V

\begin{tabular}{|l|c|c|c|c|}
\hline \multicolumn{1}{|c|}{ Ports } & $\begin{array}{c}\text { Jetée principale } \\
\text { longueur et profondeur initiale }\end{array}$ & $\begin{array}{c}\text { Jetée } \\
\text { transversale }\end{array}$ & Plan d'eau & $\begin{array}{c}\text { Ouverture } \\
\text { du port }\end{array}$ \\
\hline Agadir (nouveau port) & $2300 \mathrm{~m}(-17 \mathrm{~m})$ & $600 \mathrm{~m}$ & 60 ha & SE $(-16 \mathrm{~m})$ \\
\hline Laayoune & $2100 \mathrm{~m}(-8,5 \mathrm{~m})$ & non réalisée pour le moment & 35 ha & $\mathrm{S}(-7,5 \mathrm{~m})$ \\
\hline
\end{tabular}

16. Evolution de l'ensablement au nord du port de Laayoune

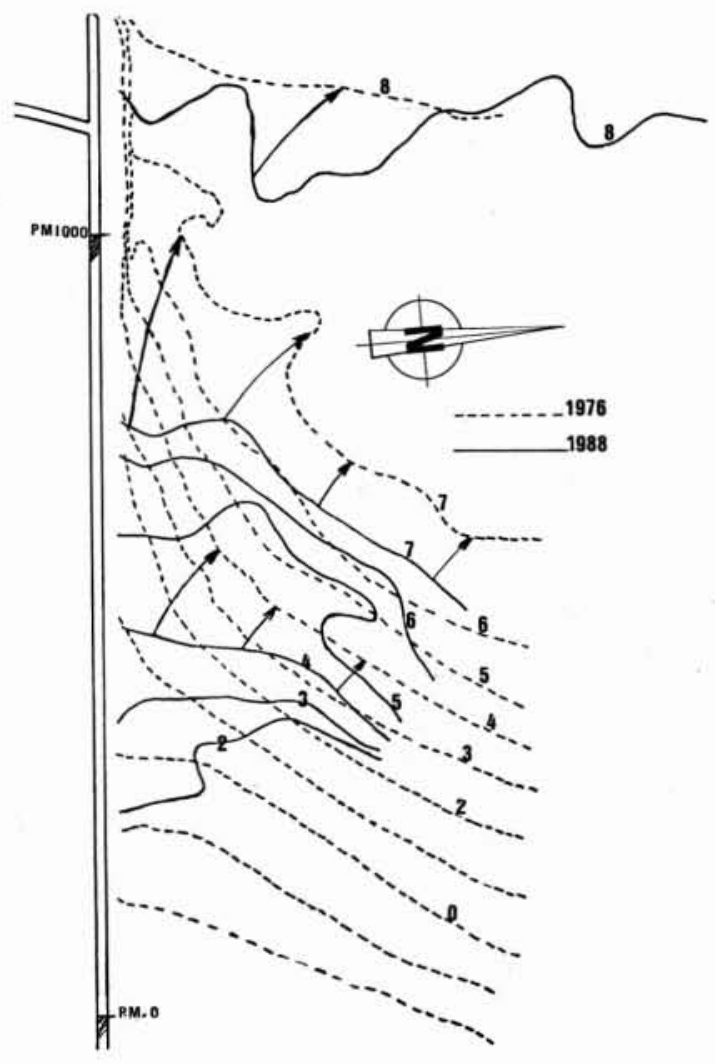

\subsection{Ports accumulant le transit littoral}

On trouve dans cette classe deux ports, le port de Laayoune (fig. 14) et le nouveau port d'Agadir (fig. 15). Leurs caractéristiques sont données dans le tableau $V$ ci-dessous :

Ces ports arrêtent pratiquement le transit littoral le long de la digue principale. La capacité totale d'emmagasinement de la plage située au vent dépendra des caractéristiques d'implantation du premier tronçon de la digue principale (longueur, profondeur, orientation, etc...), de l'orientation générale des houles dominantes, et de l'importance du transit littoral.

La capacité de saturation de la plage Nord de Laayoune a été d'environ 7 années, celle du nouveau port Agadir Anza est de 13 années après le démarrage des travaux du port. La saturation de la plage au Nord d'Anza est prévue pour 1996.
La capacité totale d'emmagasinement de la plage située au vent correspondra à peu près au volume du réceptacle limité par la crête des houles dominantes qui vient tangenter la digue dans sa partie extrême. Cependant les problèmes de contournement de la digue par les sédiments commencent à devenir importants dès que les fonds au coude atteignent des valeurs de $-5 \mathrm{~m}$ à $-7 \mathrm{~m}$ hydro. A partir de cette période, les risques d'ensablement du port et la formation de la flèche à l'extrémité de la digue principale dépendent des caractéristiques du tronçon terminal de cette digue, de l'obliquité des houles dominantes par rapport à ce tronçon, de l'importance du transit et de son orientation.

A titre de comparaison, signalons que la vitesse de propagation de la plage au vent, le long du brise-lames de Laayoune (fig. 16) est double de celui que l'on peut évaluer le long du brise-lames du port Anza d'Agadir. 
Cette propagation atteint 300 à $400 \mathrm{~m}$ par an à Laayoune et 100 à $150 \mathrm{~m}$ par an à Agadir (fig. 17).

Ces taux restent élevés comparés à ceux du port de Cotonou ( 50 à $75 \mathrm{~m} / \mathrm{an}$ ) malgré l'importance du transit littoral dans ce dernier cas (1 000000 à $\left.1500000 \mathrm{~m}^{3} / \mathrm{an}\right)$.

Après saturation de la plage au vent et contournement de la jetée, la nature et l'orientation de la flèche qui se forme en extrémité de l'ouvrage sont liées au transit « saturé " ou " sous alimenté » et aux phénomènes de diffraction et de réfraction autour des musoirs.

Les risques d'ensablement du port dépendent également du débordement de la digue principale sur la passe d'entrée, débordement qui risque de créer le long de la digue secondaire un courant dû à l'expansion latérale de la houle.

Il faut préciser que le bassin portuaire de Laayoune, en l'absence de traverse, faisait l'objet, avant même la saturation de la plage Nord, d'une sédimentation due aux transports éoliens et aux apports du courant d'expansion latérale de la houle.

Les solutions pour différer les risques d'ensablement du port sont nombreuses :
- réaliser un tenon sur la face externe de la digue (de préférence dans la direction de propagation des houles dominantes) pour augmenter la capacité d'emmagasinement de la plage située au vent. Un tenon de $250 \mathrm{~m}$ de longueur orienté dans la direction des houles dominantes différera la date de formation de la flèche de 10 années à Agadir (épi réalisé peu de temps avant la saturation de la plage Nord), et 5 années seulement dans le cas du Port de Laayoune ;

- arrêter le transit littoral par des ouvrages à l'amont du port (épi ou brise-lames) ;

- rétablir artificiellement le transit littoral de l'amont vers l'aval du port par dragage avec creusement de fosse de garde ou par un système de by-pass.

Cette dernière solution permettra d'alimenter la plage sous le vent (sous alimentée) et évitera la réalisation d'ouvrages importants de protection pour diminuer l'ensablement du port.

Cette solution est également avantageuse dans les sites où les transports éoliens sont importants.
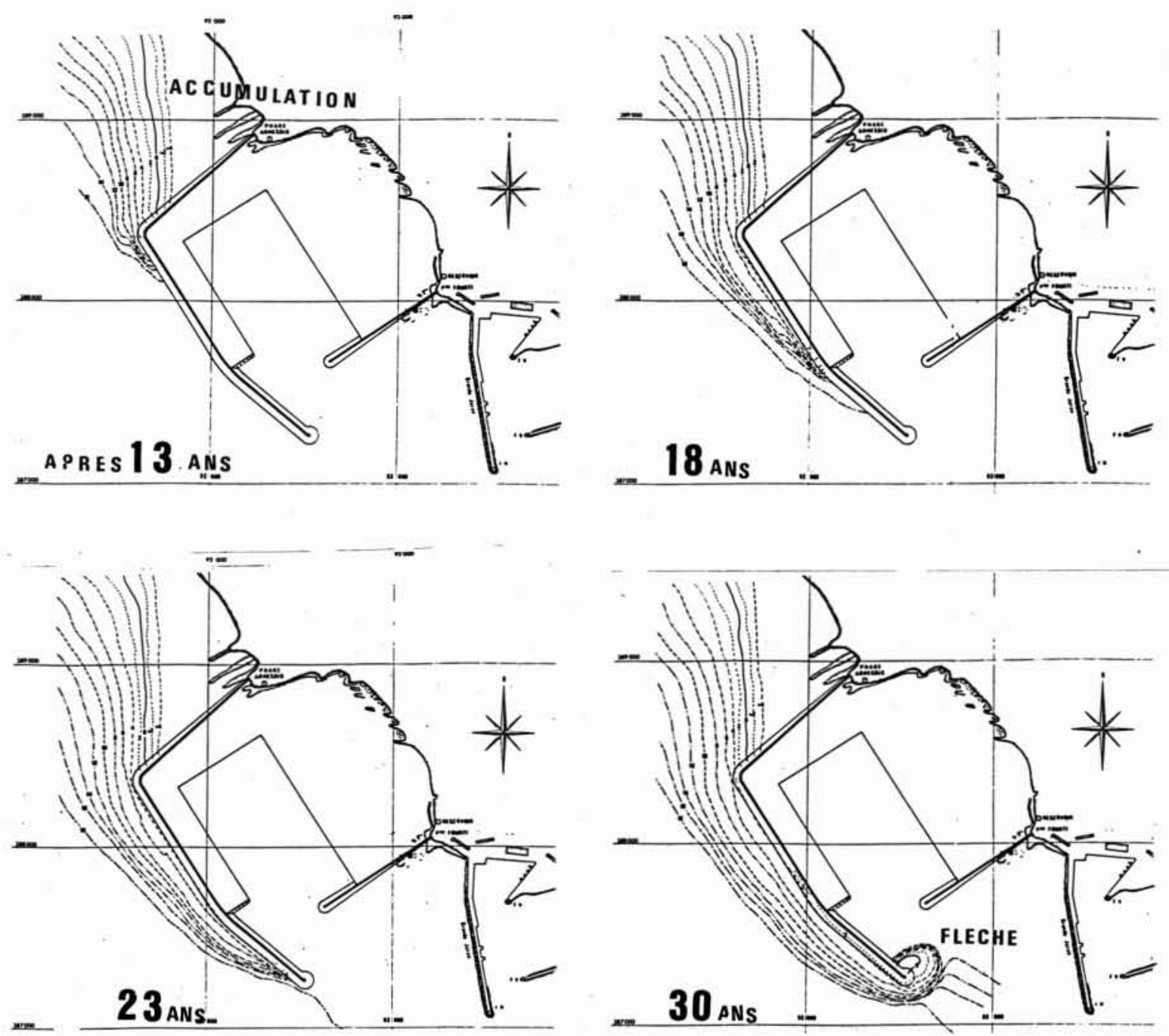

17. Prévisions de l'évolution des fonds le long de la jetée du nouveau port d'Agadir-Anza 

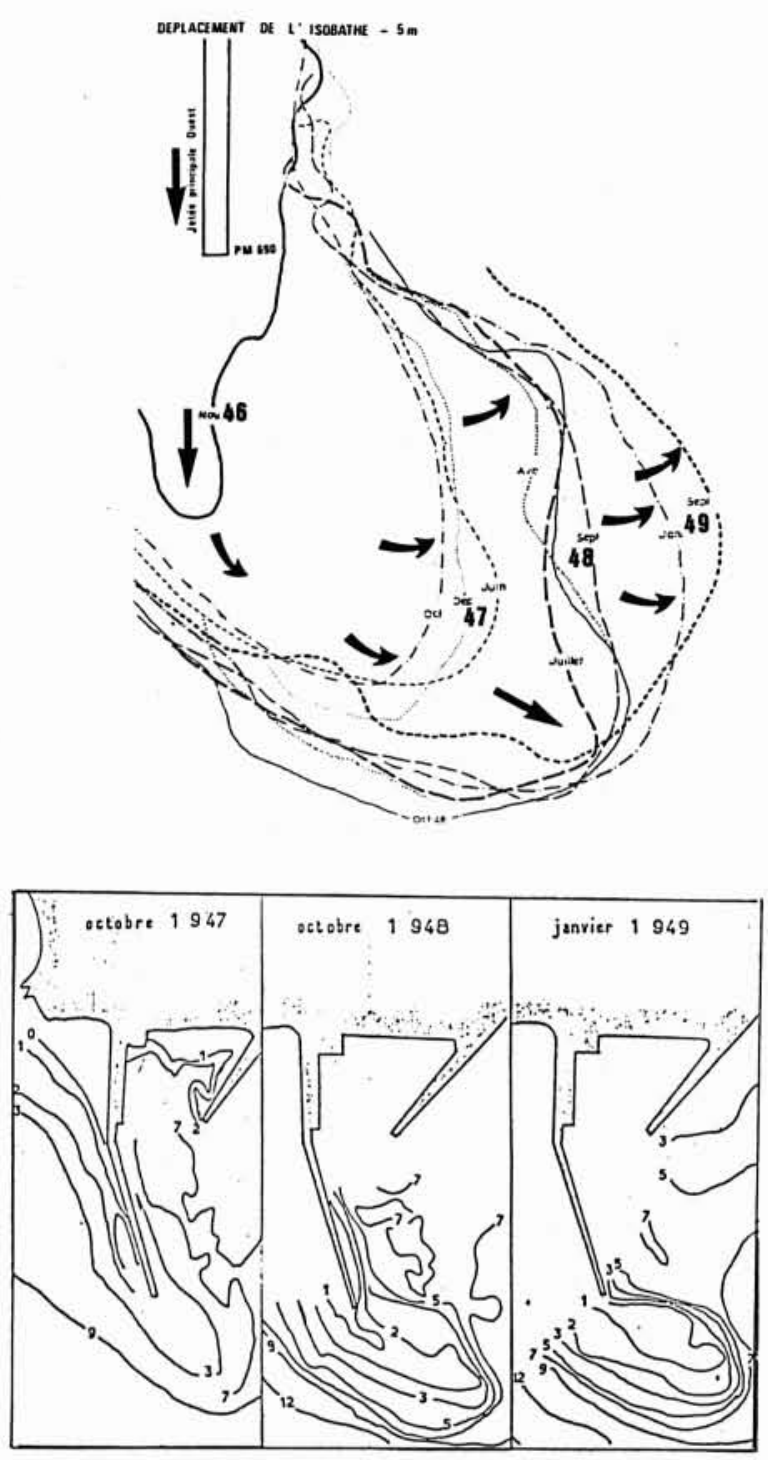

18. Formation d'une flèche sédimentaire en extrémité de la jetée principale de l'ancien port d'Agadir entre 1946 et 1949

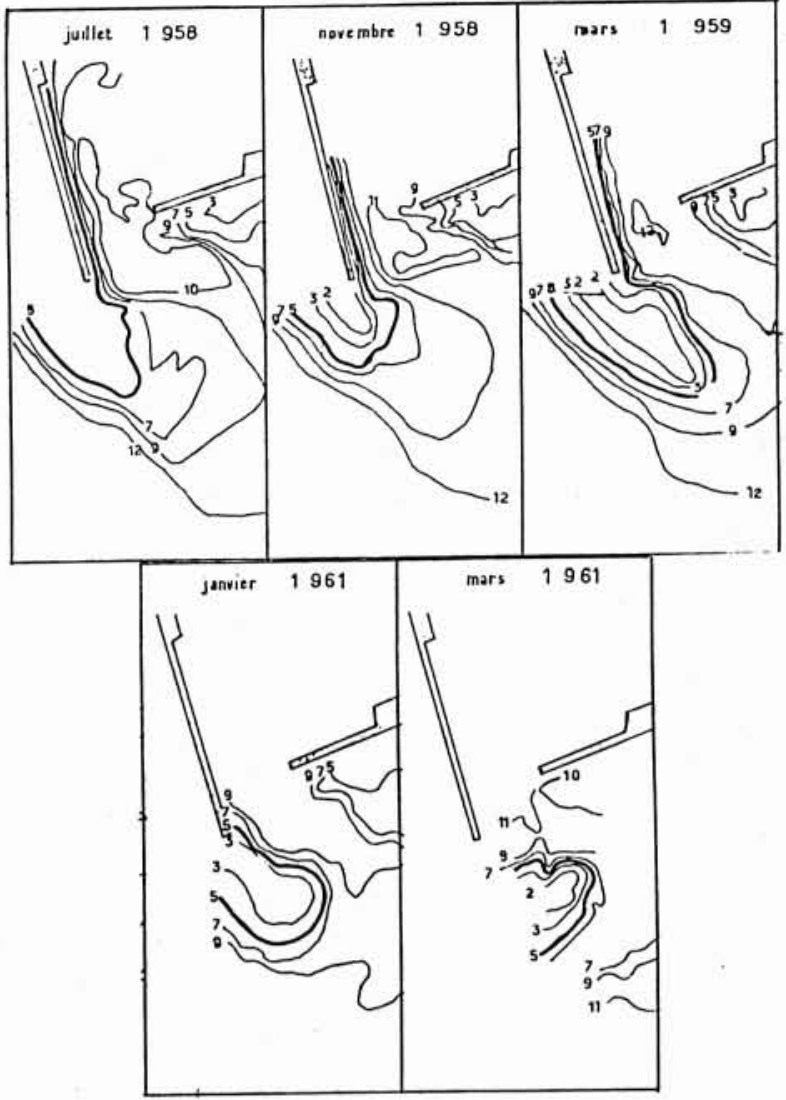

19. Evolution de la flèche entre 1958 et 1961

\subsection{Formation de flèche à l'extrémité d'une digue implantée par grandes profondeurs}

L'étude de l'évolution des fonds en extrémité de la jetée principale du port ancien d'Agadir avait montré l'apparition d'une flèche sédimentaire après le prolongement de l'ouvrage au-delà de l'isobathe $-5 \mathrm{~m}$ (musoir jetée nordsud au PM $690 \mathrm{~m}$ ).

Les études en nature et sur modèle réduit (fig. 18) avaient montré que les sables fins (diamètre moyen 0,15 à $0,20 \mathrm{~mm}$ ) étaient entraînés en suspension par les courants de houles le long de l'ouvrage et se déposaient dans un premier stade dans le prolongement de l'ouvrage. Dès que l'exhaussement des fonds avait atteint une certaine valeur ( -3 à $-4 \mathrm{~m}$ environ), on observait une modification complète dans la répartition des courants de houles qui se trouvaient rabattus en extrémité de l'ouvrage dans le sens de propagation des houles dominantes, alors qu'initialement ils restaient orientés dans le sens général de la digue. Parallèlement, les houles se réfractaient sur les hauts fonds et la flèche amorçait un pivotement vers l'intérieur du port avec une face en pente douce vers le large et très accentuée vers l'intérieur du port où les dépôts étaient limités par une ligne passant par l'extrémité de la digue et faisant un angle d'environ 50 à $60^{\circ}$ avec cette dernière (flèche saturée).

Au cours des houles faibles à moyennes, les sables au lieu de longer la digue extérieure pouvaient s'accumuler vers son enracinement. Le transit sur la flèche étant sousalimenté un pivotement apparaissait, les sédiments venant barrer l'entrée du port (fig. 19). 


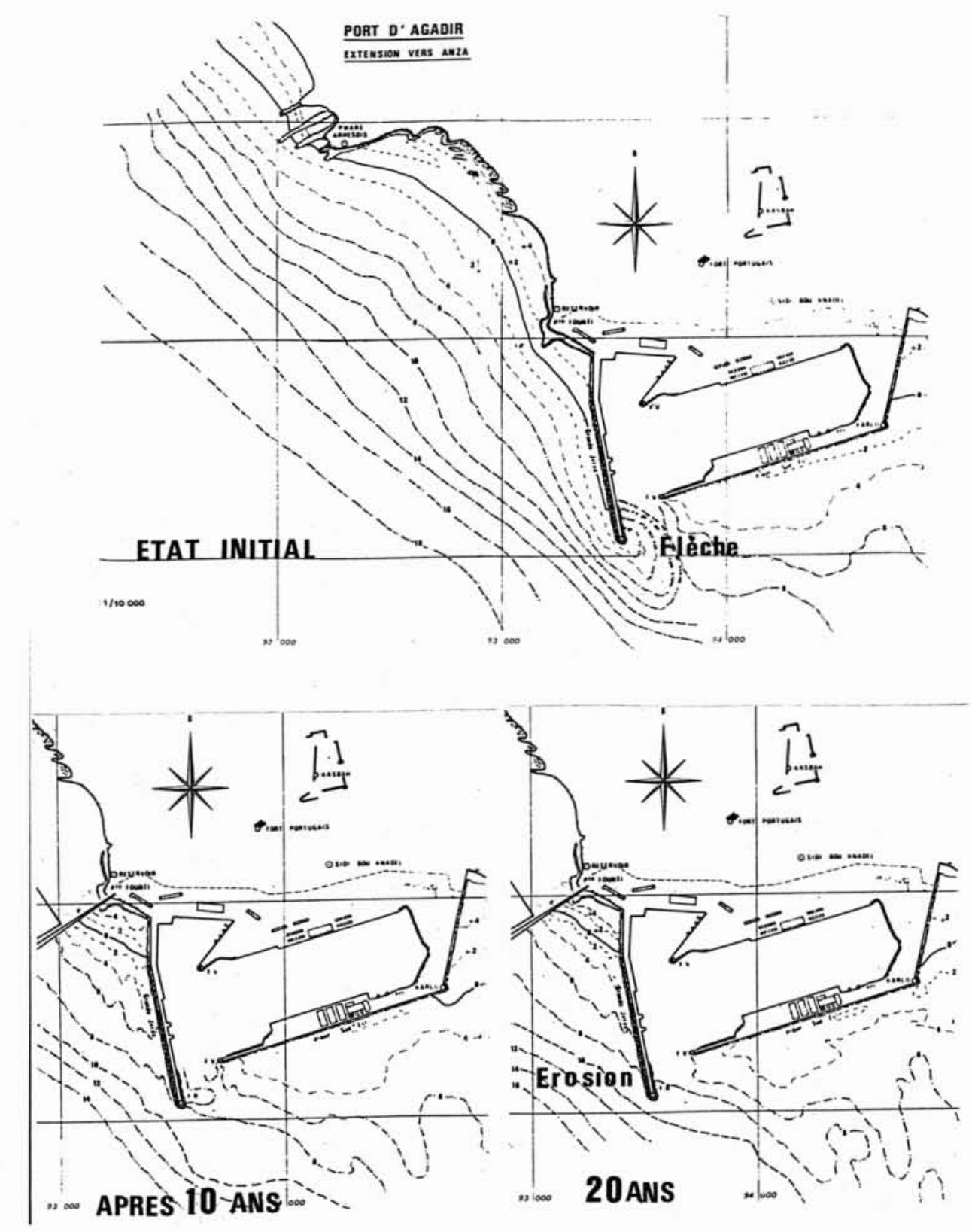

20. Disparition de la flèche sableuse après construction du nouveau port d'Agadir-Anza

Il s'est avéré possible d'assurer les accès au port ancien d'Agadir au cours de toute l'année uniquement par des dragages d'entretien intermittents en s'imposant d'étendre les dragages aux secteurs d'extrémité et à l'Ouest de la jetée principale (jusqu'à une perpendiculaire à l'extrémité de l'ouvrage). La quantité draguée a varié entre 350000 à $400000 \mathrm{~m}^{3} / \mathrm{an}$.

En l'absence de dragage, la passe d'entrée du port se trouvait entièrement bouchée et la flèche rabattue de $90^{\circ}$ par rajpport à l'alignement de la jetée principale.
Il est à remarquer que le creusement d'une fosse de garde à l'extrémité Ouest de la jetée permet de retarder la formation et le développement de la flèche. Cette solution met en œuvre des cubes de dragages importants (2,5 à 3 fois le volume du transit annuel).

La réalisation du nouveau port d'Agadir a supprimé les apports sédimentaires le long de la digue du port ancien depuis 1985. La flèche a disparu complètement en fin 1987 (fig. 20).

\section{LA HOUILLE BLANCHE/ $/ \mathrm{N}^{\circ} 1-1991$}


7. Ports implantés par profondeurs moyennes ( -5 à - $7 \mathrm{~m}$ ) dans la zone de la barre

On peut classer dans cette catégorie les nouveaux ports marocains de Sidi Ifni (fig. 21), Tan-Tan (fig. 22 et 23) et Tarfaya (fig. 24 et 25). Les caractéristiques principales sont données dans le tableau VI.

Les ouvrages précités sont implantés dans la zone voisine de la barre, le long d'une côte soumise à d'importants remaniements sédimentaires et orientés en sens contraire du transit littoral dominant (ouverture des ports côté Sud). Ils posent des problèmes délicats d'ensablement par suite de leur intéraction sur les mouvements sédimentaires.
Ces ouvrages n'arrêtent qu'une partie du transit littoral, les processus de fonctionnement étant liés à la hauteur des houles et à l'importance du transit. En houles de tempêtes et moyennes une grande partie des sables est déviée et vient augmenter le débit solide dans la barre et en houles faibles les apports peuvent être stoppés le long de l'ouvrage.

Le contournement des sédiments par l'ouvrage de protection principale est important et peut varier aux environs de 50 à $100 \%$.

Plages au nord des ports: La présence des platiers rocheux limite considérablement les accumulations sur ces plages par rapport à leur capacité d'emmagasinement. Pour les ports de Tan-Tan et Tarfaya la saturation a été atteinte avant 1982, soit 1 à 2 années après l'achèvement des travaux des ports (1977-1979).

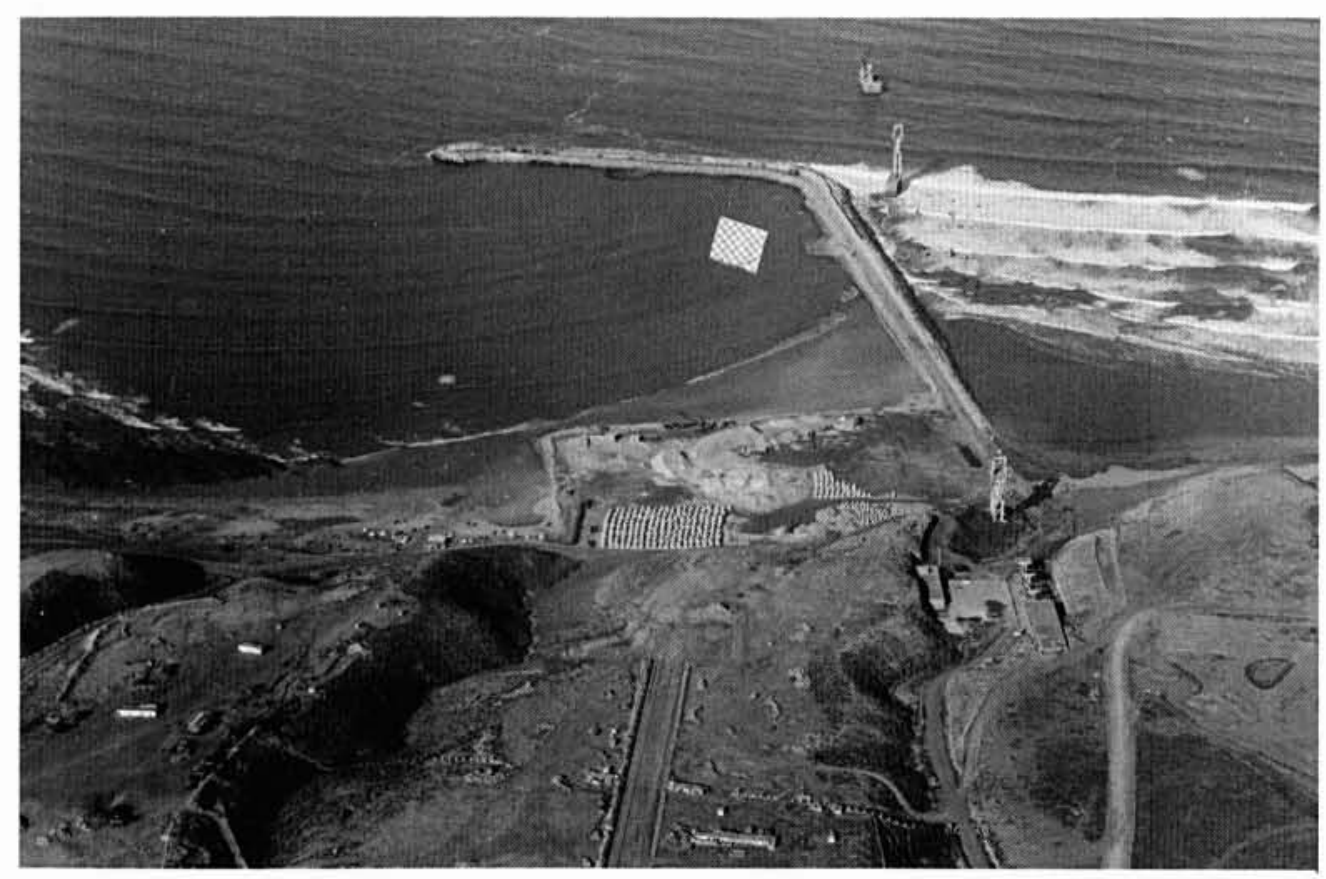

21. Port de Sidi-Ifni en cours de construction

Tableau VI

\begin{tabular}{|c|c|c|c|c|}
\hline Ports & $\begin{array}{c}\text { Longueur jetée principale (*) } \\
\text { profondeur }\end{array}$ & Jetée transversale (*) & Houles dominantes & Transit littoral \\
\hline Tan-Tan & $\begin{array}{c}1923 \mathrm{~m}(-8 \mathrm{~m}) \\
\text { épi }: 600 \mathrm{~m}(-2 \mathrm{~m})\end{array}$ & $548 \mathrm{~m}(-5,5 \mathrm{~m})$ & $290^{\circ}-320^{\circ}$ & $450000 \mathrm{~m}^{3} / \mathrm{an}$ \\
\hline Tarfaya & $\begin{array}{c}1140 \mathrm{~m}(-6 \mathrm{~m}) \\
\text { épi }: 550 \mathrm{~m}(-4 \mathrm{~m})\end{array}$ & $270 \mathrm{~m}(-2 \mathrm{~m})$ & NNW-N & $400000 \mathrm{~m}^{3} / \mathrm{an}$ \\
\hline Sidi Ifni & $\begin{array}{c}1140 \mathrm{~m}(-8 \mathrm{~m}) \\
\text { épi : } 650 \mathrm{~m}(-6,5 \mathrm{~m})\end{array}$ & non réalisé & WNW à $\mathrm{N}$ & $400000 \mathrm{~m}^{3} / \mathrm{an}$ \\
\hline
\end{tabular}

(*) Profondeurs avant implantation des ouvrages. 
Dans le cas du port de Sidi Ifni, les accumulations côté Nord restent également limitées à cause des platiers rocheux et du fait que le site est soumis à des houles ayant de grandes différences d'amplitude, de période, et de direction, qui favorisent la déperdition des sédiments vers le large.

Flèche sédimentaire à l'extrémité de l'ouvrage principale de protection: Les ouvrages implantés dans la zone de la barre connaissent peu de temps après leur réalisation la formation d'une flèche importante à leur extrémité dont le développement et les caractéristiques dépendent du transit littoral contournant la digue, des caractéristiques du tronçon terminal de la digue principale et de l'orientaion des houles dominantes (fig. 26).
Dans le cas du port de Tan-Tan où le brise-lames est disposé parallèlement à la côte, la flèche se forme au départ dans l'alignement de l'ouvrage et se rabat quelques temps plus tard. Après exhaussement suffisant des fonds vers le chenal d'accès au port dans le sens de propagation des houles dominantes, elle continue à se développer avec une direction à environ $55^{\circ}$ par rapport à l'alignement du brise-lames.

Dans le cas du port de Tarfaya où le dernier tronçon du brise-lames est incliné par rapport à la ligne du rivage, il a été constaté que la flèche se forme et se développe dans l'alignement du dernier tronçon du brise-lames et ne change pas de direction.

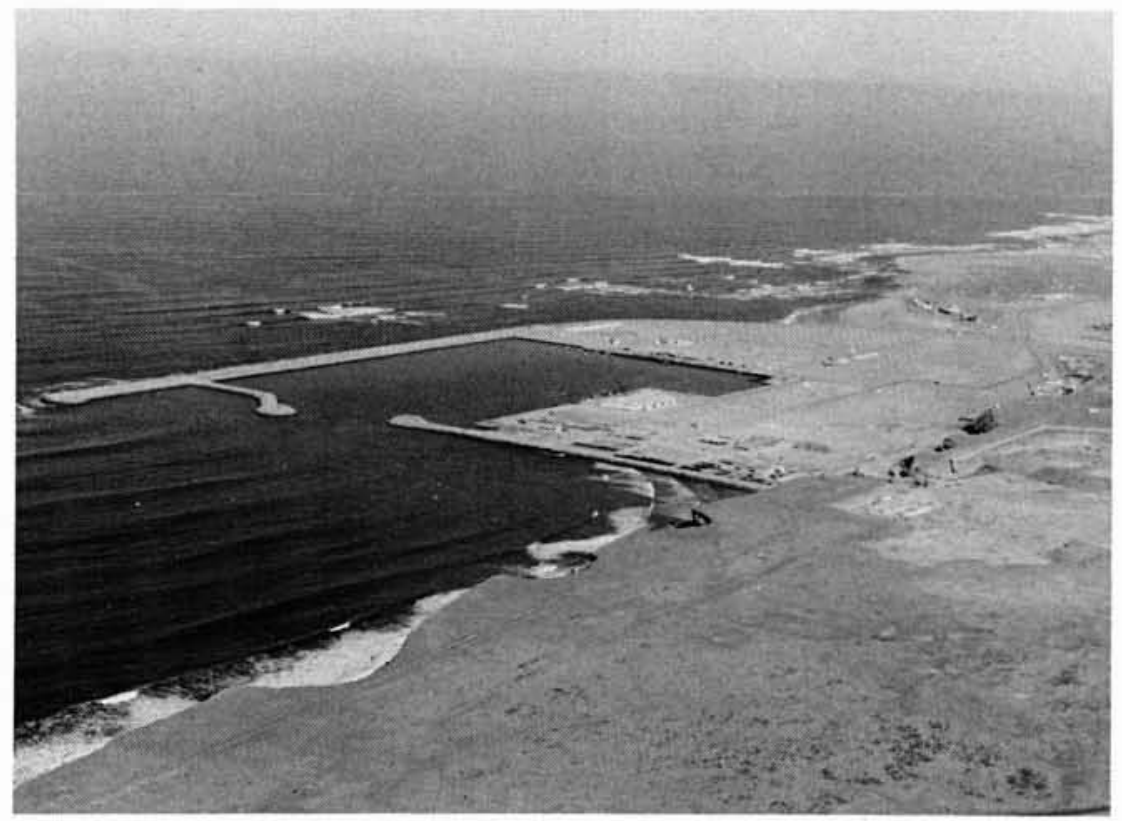

22. Port de Tan-Tan en 1984

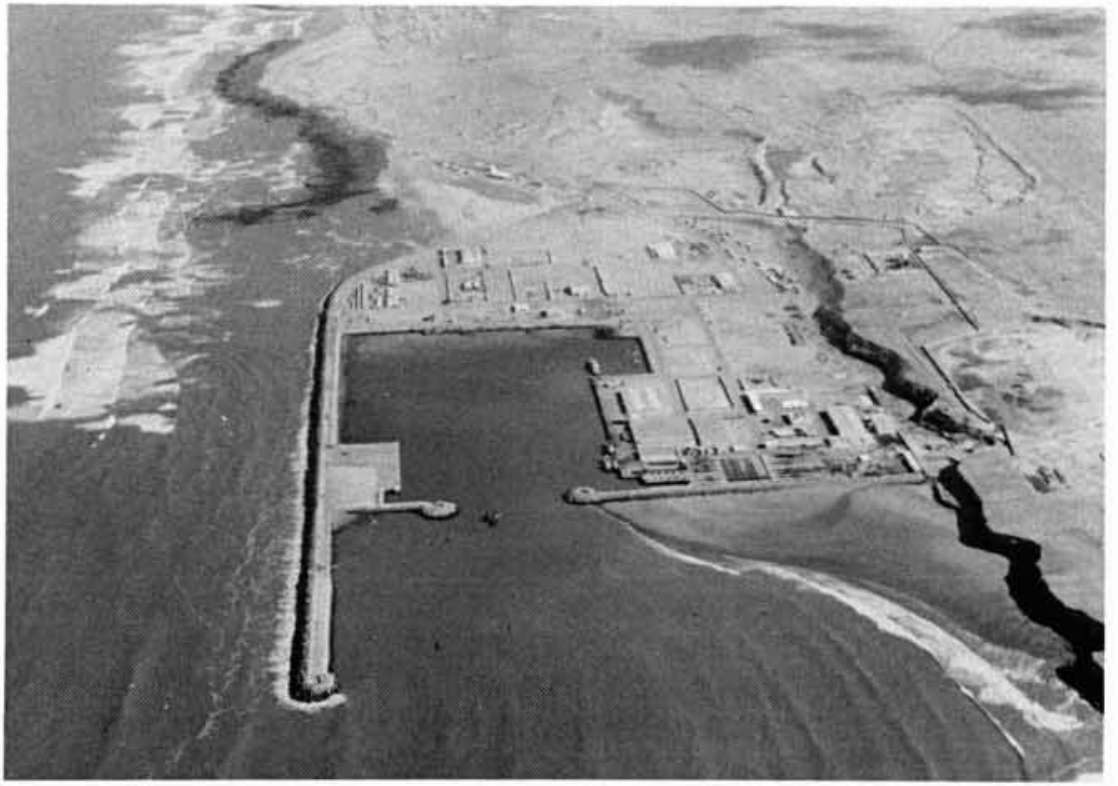

23. Port de Tan-Tan en 1988 


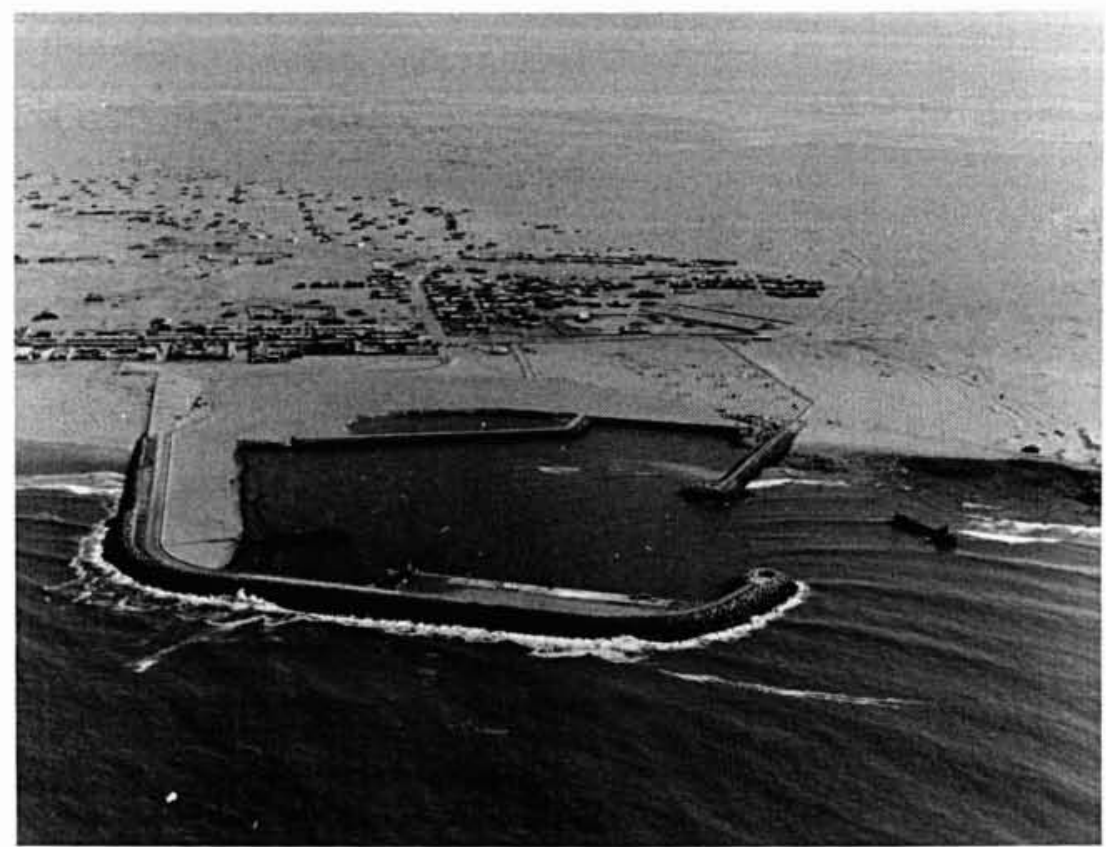

24. Port de Tarbaya en 1984

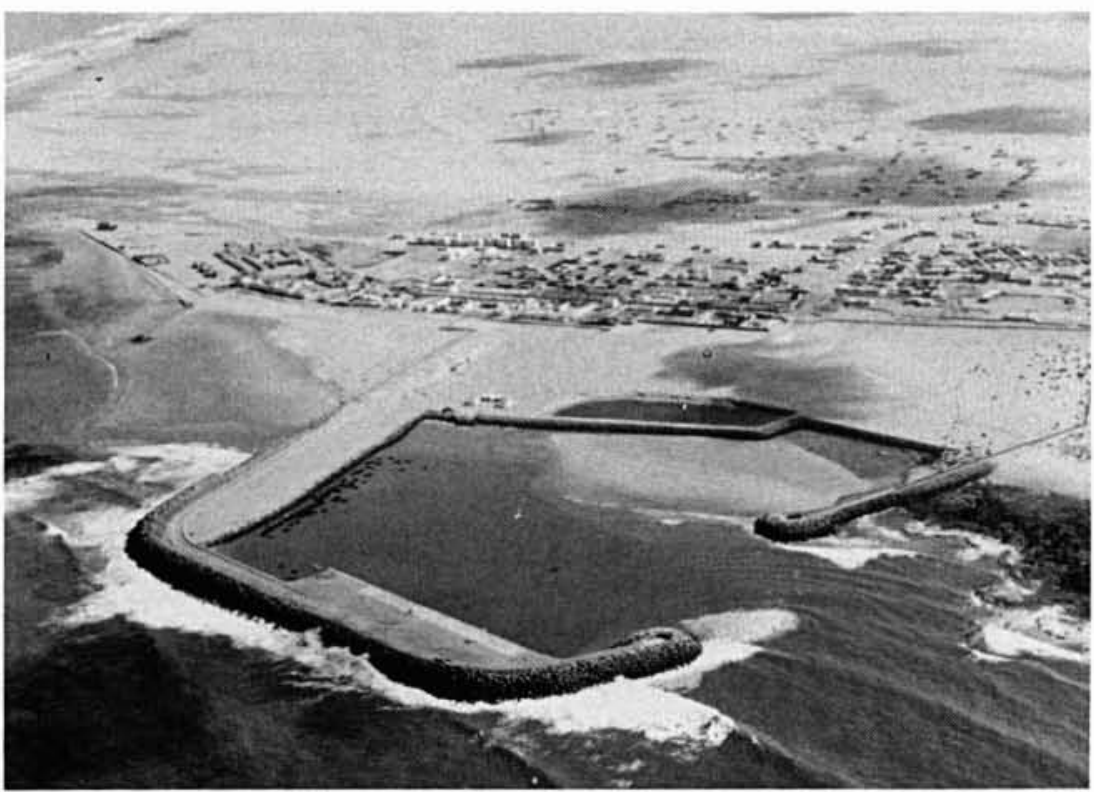

25. Port de Tarbaya en 1988

DIFFERENTS TYPES DE FLECHES

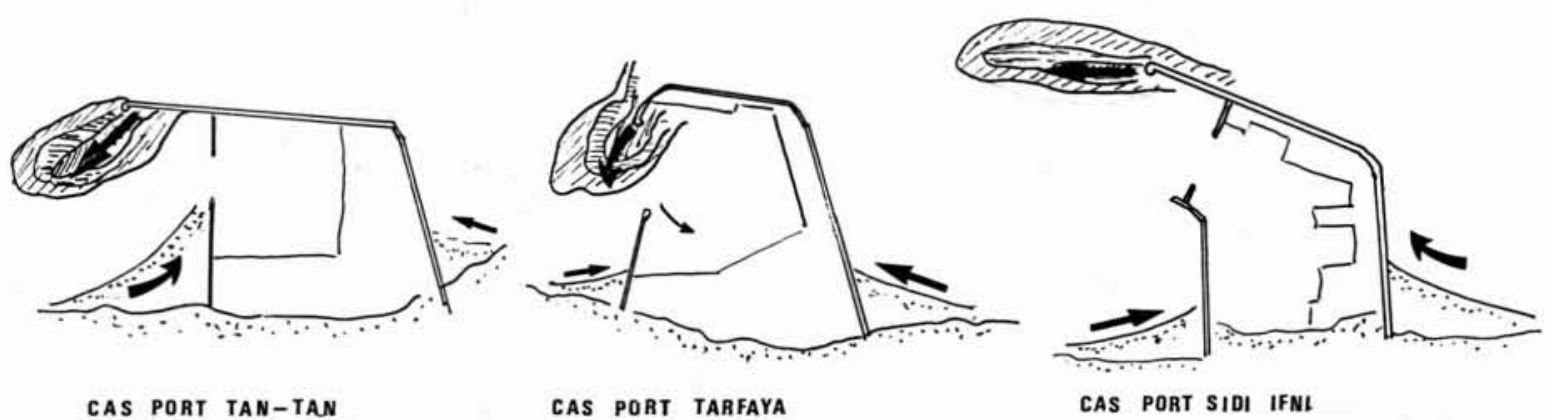

26. Les dépôts sédimentaires en fonction de l'implantation des ouvrages 

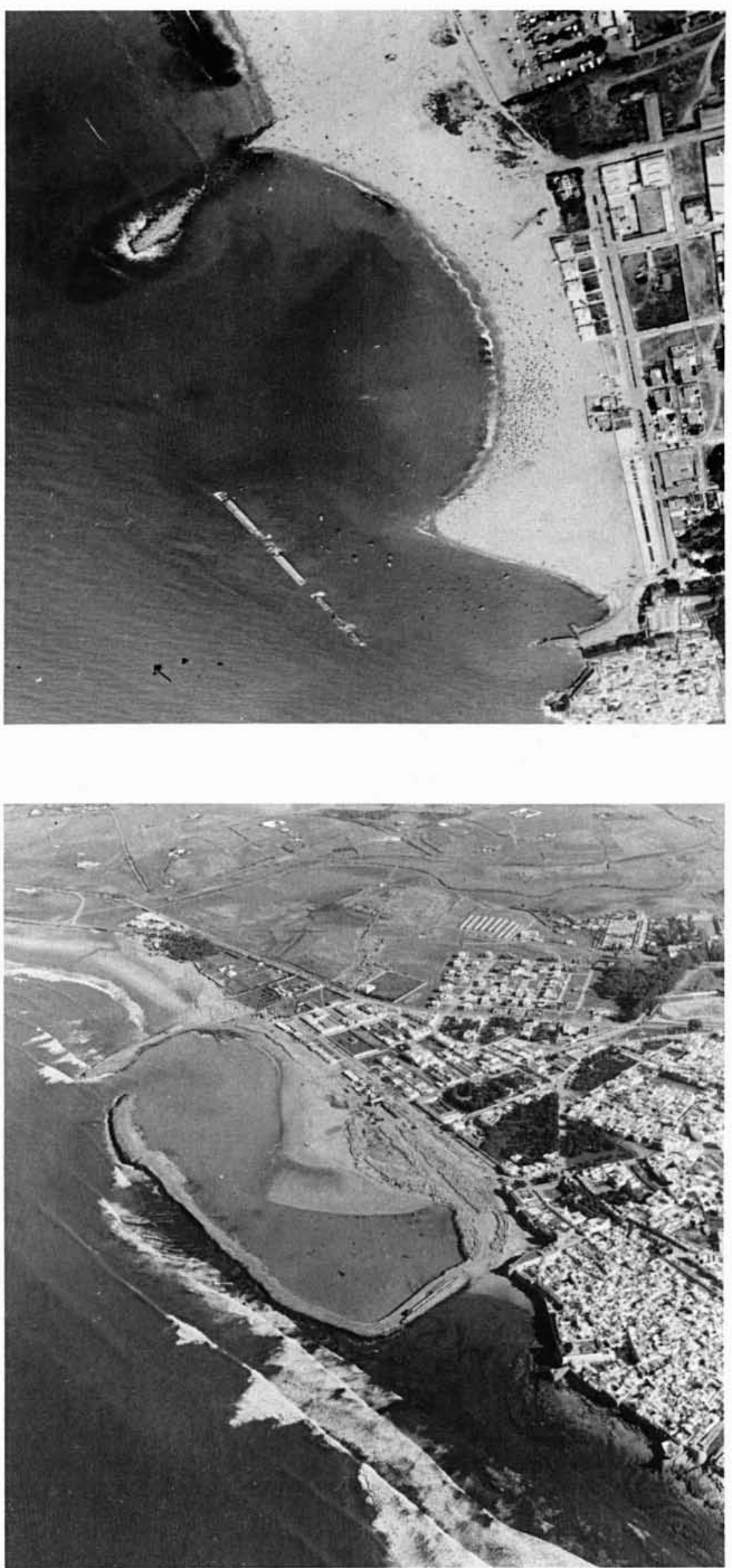

27 et 28. Port d'Asilah 
Plage adossée à la traverse ou digue secondaire: Les dépôts sur cette plage proviennent principalement des apports du courant d'expansion latérale de la houle dans le cas où les digues de protection débordent sur les passes d'entrée aux ports

Le transit littoral de sens opposé au transit principal Nord Sud, en général faible, contribue peu à l'ensablement. mais un phénomène de "piégeage " de ces apports peut se produire. Après saturation de la plage, les sédiments peuvent atteindre la passe et la colmater.

Au port de Tan-Tan, où la longueur de recouvrement est importante, les taux de comblement de la plage ont des valeurs de :

- $40000 \mathrm{~m}^{3} / \mathrm{an}$ en moyenne avant le prolongement de la jetée principale ;

- $75000 \mathrm{~m}^{3} /$ an après le prolongement de la jetée précitée sur $225 \mathrm{~m}$.

Il semble que le taux de comblement de la plage adossée à la traverse a doublé avec le doublement de la longueur de recouvrement.

Au port de Sidi Ifni, les apports par le courant d'expansion latérale de la houle et ceux du transit littoral (SN) provoquent, en l'absence de la traverse, une sédimentation importante du bassin portuaire avec formation d'un terreplein à l'enracinement de la jetée principale côté Sud, terre-plein qui progresse en l'absence de dragages vers le môle central. Ces apports augmenteront au fur et à mesure du contournement de la jetée principale par les sables et seront d'autant plus importants que la jetée transversale ne sera pas réalisée.

Passe d'entrée et bassin portuaire: Dans les deux ports de Tan-Tan et Tarfaya, il a été constaté que les fonds évoluent très vite dans les chenaux et passes d'entrée. Cependant les fonds dans les bassins portuaires des ports précités n'évoluent que peu, exception faite de la partie Sud du bassin de Tarfaya, transformée en terre-plein par les atterrissements de sable. Il reste à préciser la contribution importante des apports éoliens dans la sédimentation du bassin portuaire de Tarfaya (environ $80000 \mathrm{~m}^{3} / \mathrm{an}$ ).

Littoral avoisinant les ports :

Il est important de remarquer, pour les deux ports TanTan et Tarfaya, que :

- les fonds au large des ports côté Nord et Sud ont peu évolués, cette évolution est liée à la variation cyclique des fonds, qui semblent connaître un équilibre dynamique. Cependant les fonds au large immédiat du brise-lames ont connu un engraissement important ;

- les fonds au Sud immédiat des ports dans le prolongement de l'emprise de ceux-ci ont connu également une sédimentation importante dans la zone du courant d'expansion latérale de la houle.

Dans le cas des ports précités de la côte Sud du Maroc, il est pratiquement impossible d'envisager une maintenance des profondeurs du chenal d'accès et de la passe d'entrée par des dragages classiques, car une simple tempête suffira à colmater les fonds en quelques jours. Aussi les solutions pour améliorer l'exploitation des ports précités consisteraient à diminuer ou arrêter le transit littoral dans la zone au vent. Pour ce faire les solutions suivantes sont envisageables :

- prolongation du $1^{\mathrm{er}}$ tronçon de la digue principale formant épi à des profondeurs plus importantes et de préférence englobant la zone des brisants. La direction de prolongation dépendra des caractéristiques des houles dominantes ;

- réalisation d'un brise-lames ou d'un épi dans la zone amont pour réduire le transit littoral devant l'ouvrage et de ce fait abaisser la côte de la barre à une valeur compatible avec celle des bâteaux fréquentant le port.

Ces solutions peuvent être dimensionnées pour éviter une brusque obstruction des accès du port et la formation brutale de la flèche aux extrémités des ouvrages de protection étant entendu que des dragages d'entretien resteront nécessaires pour maintenir les fonds dans les ports et leurs accès.

Pour éviter la saturation des ouvrages de protection, il faudrait extraire soit par dragages, soit par une installation de by-pass les matériaux déposés dans la zone au vent et les refouler dans les zones sous le vent à des distances suffisantes pour éviter leur reprise par le courant d'expansion latérale de la houle. Un épi de protection, délimitant ce secteur, peut être nécessaire pour supprimer un tel phénomène.

\section{Ouvrages implantés près du littoral par des petites profondeurs (inférieures à $-\mathbf{3} \mathbf{m}$ )}

Ces ouvrages agissent très peu sur le transit littoral dans la barre, une grande partie des sédiments cheminant devant les aménagements portuaires quels qu'ils soient. Les fonds situés de part et d'autre de l'aménagement en question sont peu modifiés. Les accumulations qui risquent d'ensabler ces ouvrages résultent d'un transport d'estran ou de la seule action de faibles houles.

Il faudra éviter par ailleurs que la protection de la passe d'entrée ne soit trop importante afin de ne pas créer une zone d'expansion de la houle propice aux apports sédimentaires. Les ports entrant dans cette classe de petits ouvrages sont nombreux sur la côte atlantique marocaine : ports de pêche ou de plaisance et prises d'eau.

Parmi les ouvrages implantés sur une côte à faible transit littoral citons les ports d'Asilah (fig. 27 et 28) et des Sables d'Or (fig. 29). La sédimentation du port d'Asilah se fait sous la forme d'un tombolo qui avance vers le brise-lames. Le taux annuel d'ensablement est inférieur à $30000 \mathrm{~m}^{3} / \mathrm{an}$, dont environ $50 \%$ sont dus à l'érosion éolienne, le reste correspond à des apports marins. Le taux de sédimentation du port des Sables d'Or reste inférieur à $15000 \mathrm{~m}^{3} / \mathrm{an}$.

Le port de Boujdour, dont le plan d'eau fait 2 ha, est situé dans une zone à forts transits sédimentaires. Cet ouvrage, depuis sa réalisation en 1982 à aujourd'hui, n'a jamais fait l'objet de dragage, et il est complètement ensablé (fig. 30). Cette sédimentation est due aux apports du courant d'expansion latérale de la houle. Le littoral avoisinant le port de Boujdour a été peu perturbé par celui-ci. En effet, les comparaisons de sondages récents avec ceux 

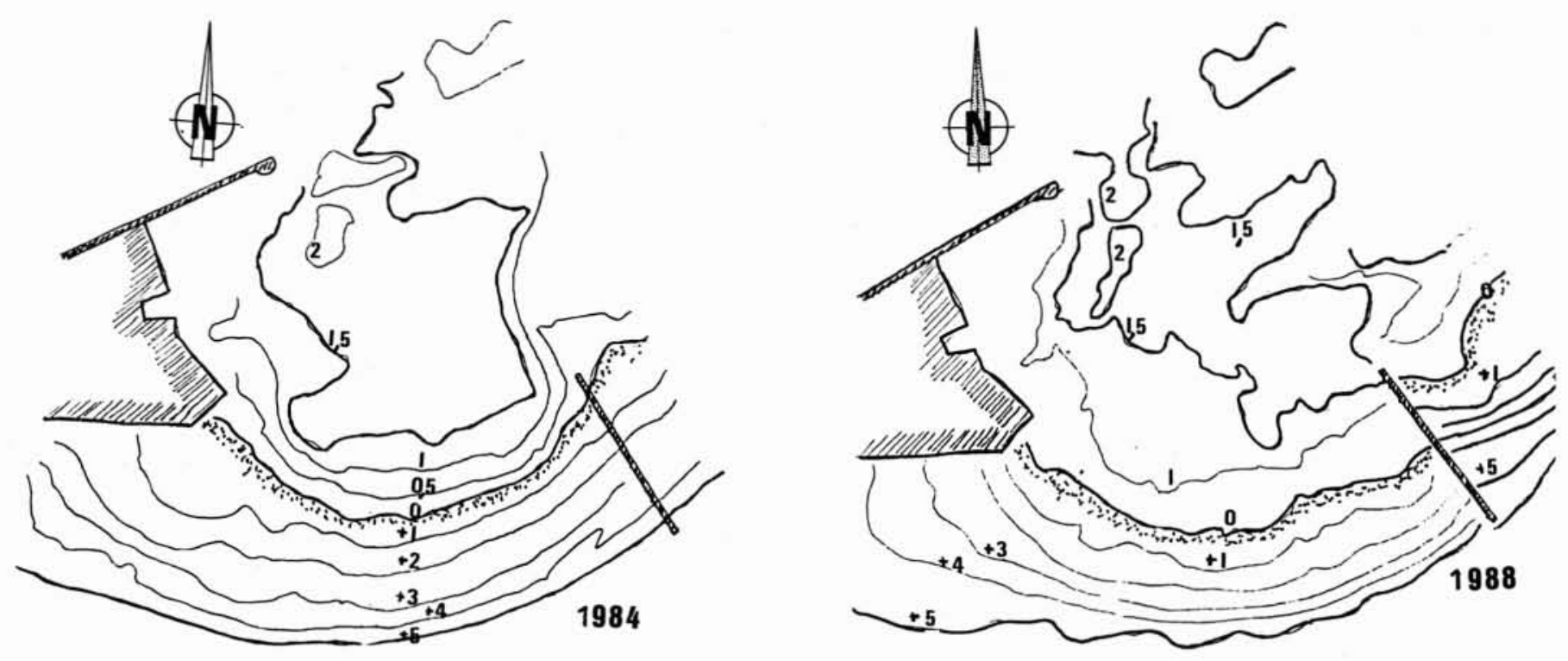

29. Le port des Sables d'Or. Etat des fonds en 1984 et 1988

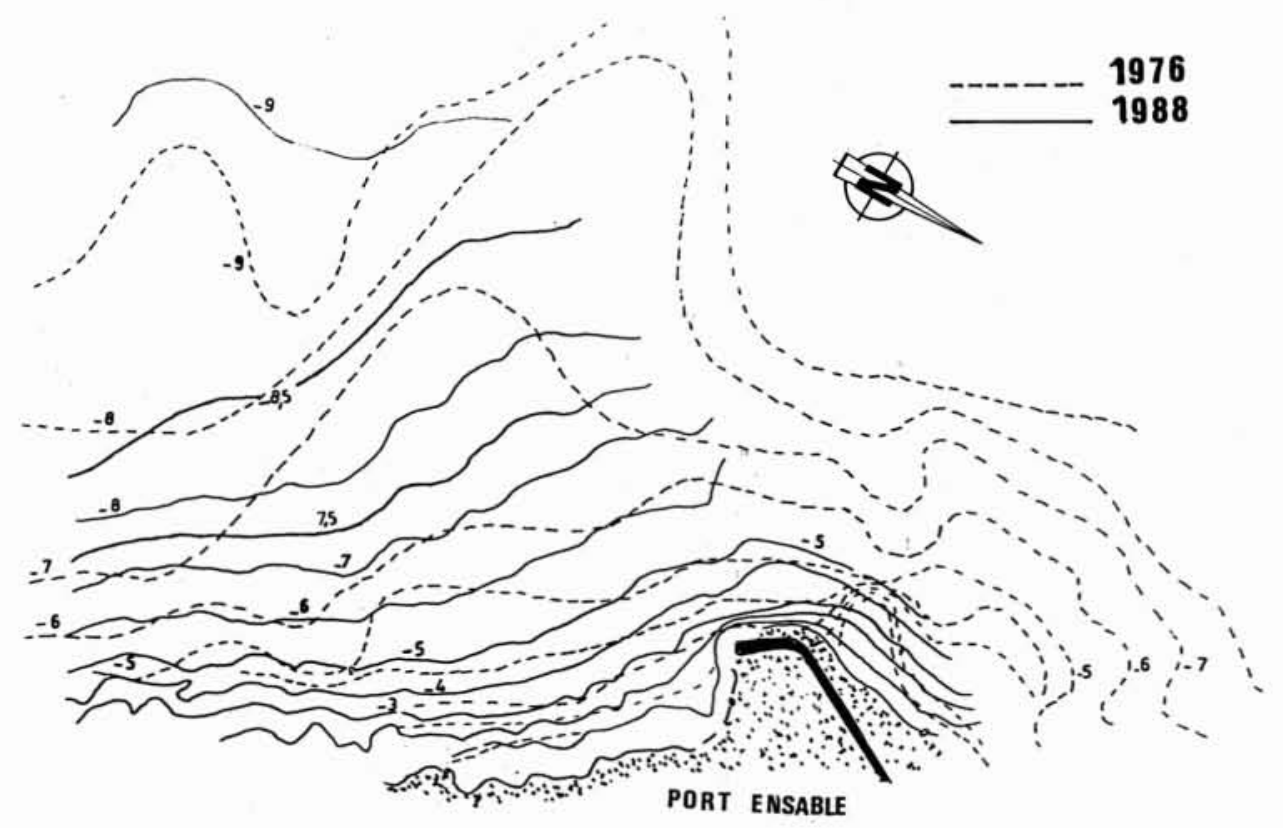

30. Ensablement du port de Boudjour 
datant avant la réalisation de l'ouvrage ont montré un léger engraissement au Nord de l'épi et un léger ensablement des fonds au large immédiat du port. Cette perturbation est arrivée actuellement à un état d'équilibre.

\section{Impact sédimentologique des réalisations portuai- res de la côte atlantique sur le littoral}

L'équilibre sédimentaire d'un littoral est très sensible à toutes les modifications qui peuvent intervenir dans son environnement, que ces modifications soient dues à des causes naturelles ou à des interventions humaines.

Il est en effet toujours recherché au niveau de la conception portuaire une perturbation minimale de la sédimentologie du site et de ses environs. Malheureusement, s'il n'est pas difficile de respecter le critère précité pour les aménagements portuaires implantés sur une côte à faibles mouvements sédimentaires, un tel respect paraît difficile et parfois onéreux sur une côte à forts transits sédimentaires.

Un ouvrage portuaire peut influencer les mouvements sédimentaires dus à la houle en :

- perturbant le transit littoral ;

- perturbant le transport de masse ;

- perturbant le transport sur la barre.

\subsection{Impact sédimentologique des ouvrages provoquant une forte expansion latérale de la houle}

On classe dans cette catégorie 3 sites :

- Baie de Tanger (zone Est);

- Plage Est de Mohammédia,

- Plage Est des Sables d'Or Nord.

Les ouvrages portuaires de Tanger, Mohammédia (port pétrolier) et Sables d'Or ont coupé la propagation des houles N.W. Les zones à l'abri des digues de protection ont constitué un piège pour les matériaux entraînés par les clapots et vents du secteur N.E. Par contre, dans les zones à l'Est des baies ou anses, le littoral n'étant plus alimenté par les sables apportés par les houles du secteur N.W. subit des érosions importantes allant jusqu'aux fonds de $-5 \mathrm{~m}$.

Le phénomène a été accentué dans la baie de Tanger par l'érosion éolienne qui représente $30 \%$ de l'érosion totale.

\begin{tabular}{|l|l|}
\hline \multicolumn{1}{|c|}{ Sites } & \multicolumn{1}{c|}{ Taux d'érosion } \\
\hline Baie de Tanger & $\begin{array}{l}\text { Oueds Mogoga et Gandori } \\
55000 \text { à } 60000 \mathrm{~m}^{3} / \mathrm{an}(2 \text { à } \\
3 \mathrm{~m} / \mathrm{an})\end{array}$ \\
\hline Anse Sables d'Or & $\begin{array}{l}\text { Secteur Est Anse } \\
10000 \text { à } 15000 \mathrm{~m}^{3} / \mathrm{an}\end{array}$ \\
\hline Baie de Mohammédia & $\begin{array}{l}\text { Plage Est }- \text { plus de } \\
50000 \mathrm{~m}^{3} / \mathrm{an}\end{array}$ \\
\hline
\end{tabular}

Les sites précités sont situés sur une côte à faible transit littoral et à forts mouvements dans le profil. Les protections de haute plage du type mur vertical en gabions réalisées à Mohammédia et Tanger se sont avérées mal adaptées à ce type de problème et n'ont pu résister aux attaques des houles de tempêtes plus d'une année. Les solutions efficaces de protection pouvant reconstituer les plages doivent être à base d'épis, brise-lames ou mixtes (épis + brise-lames).

Les études du L.C.H.F. ont permis de retenir pour la baie de Tanger une protection portant sur 4 épis (longueur 260 à $290 \mathrm{~m}+5 \mathrm{~m}$ ) et 3 brise-lames (longueur $150 \mathrm{~m}$ à $+3 \mathrm{~m}$ ). Depuis 1985, date de réalisation de la $1^{\mathrm{re}}$ tranche de la protection envisagée devant la zone urbanisée, les isobathes zéro et $-3 \mathrm{~m}$ ont avancé (en 1988) respectivement de 50 et 70 à $100 \mathrm{~m}$ (fig. 31 ).

Des mesures pour atténuer les érosions éoliennes ont été également adoptées à Tanger.

Des solutions similaires peuvent être optimisées sur modèle réduit pour la plage Est de Mohammédia et pour celle des Sables d'Or.
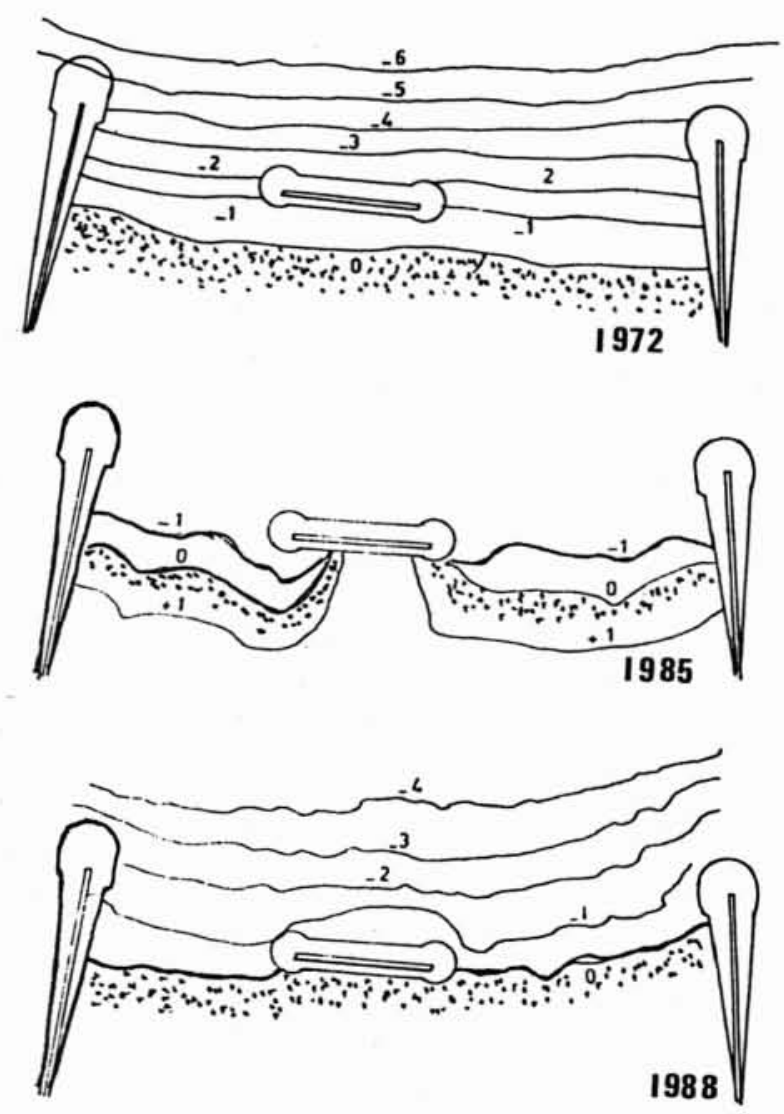

31. Protection de la plage de Tanger entre les oueds Mogoga et Kandari. Etats des fonds 


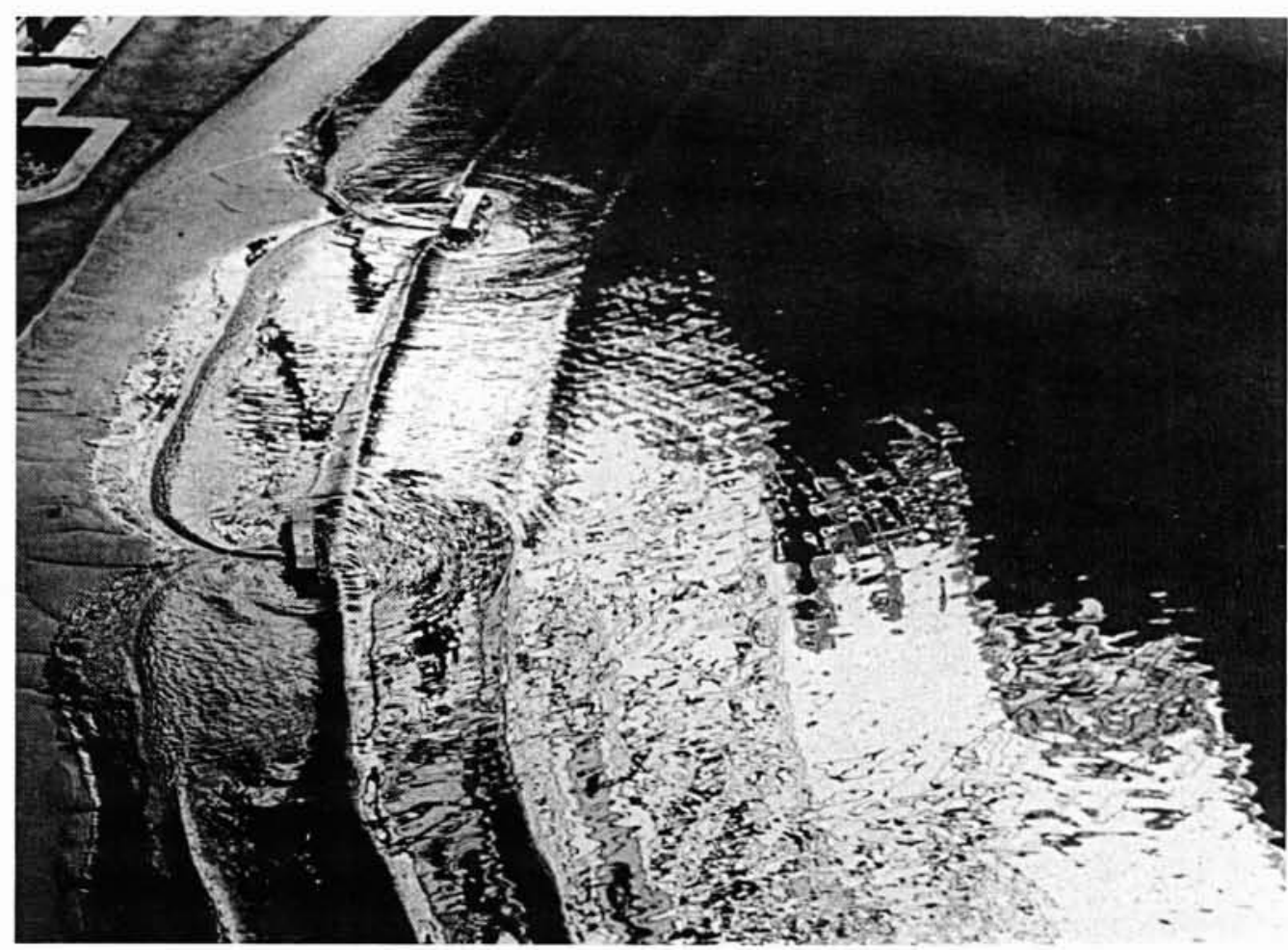

32. Etude en modèle réduit de la protection de la plage d'Agadir par des brise-lames arasés à la côte de pleine mer

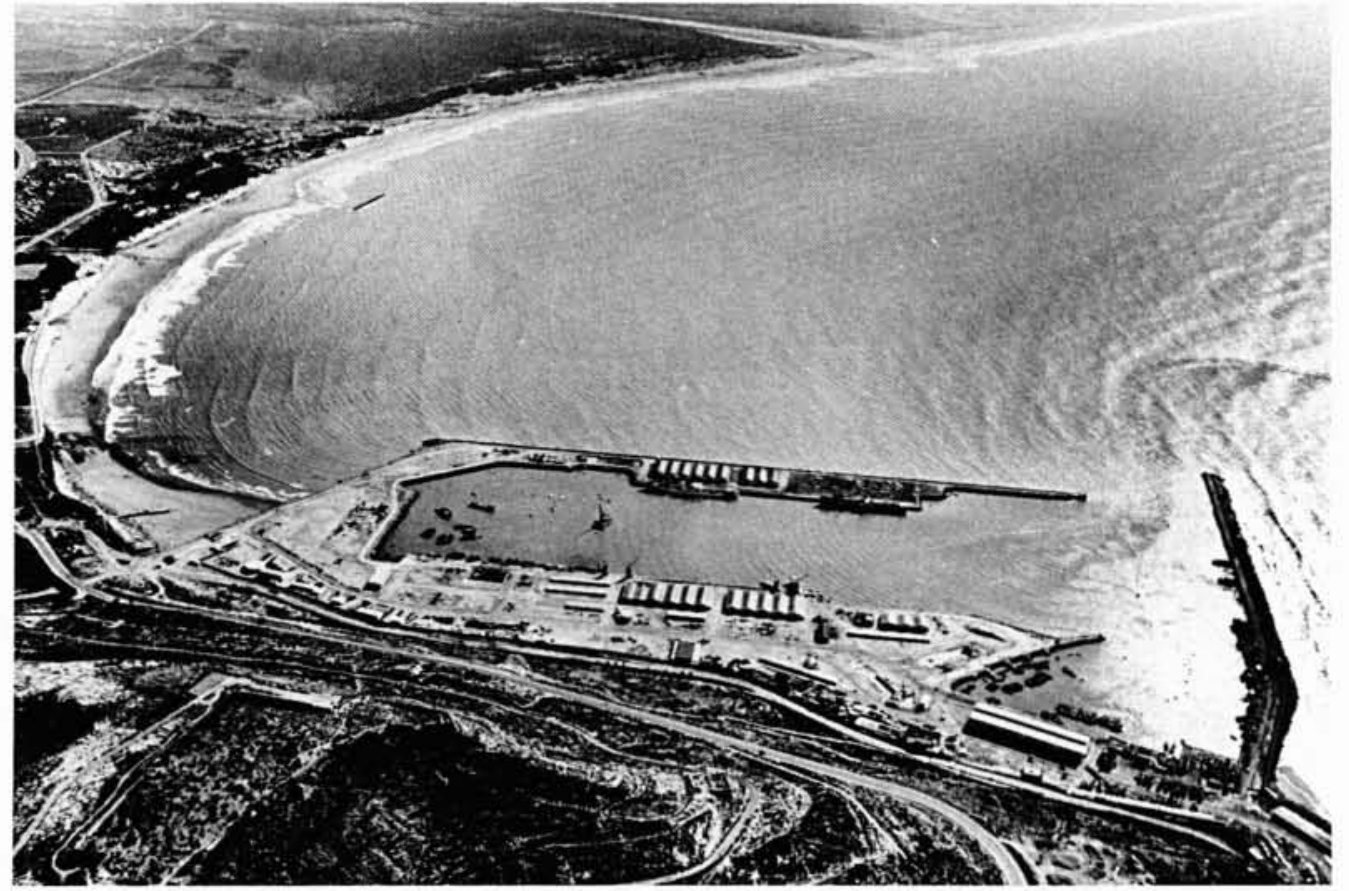

33. La plage d'Agadir reconstituée après réalisation du briselames et rechargement des petits fonds de $-2 a ̀-3 m$ par les produits de dragage 


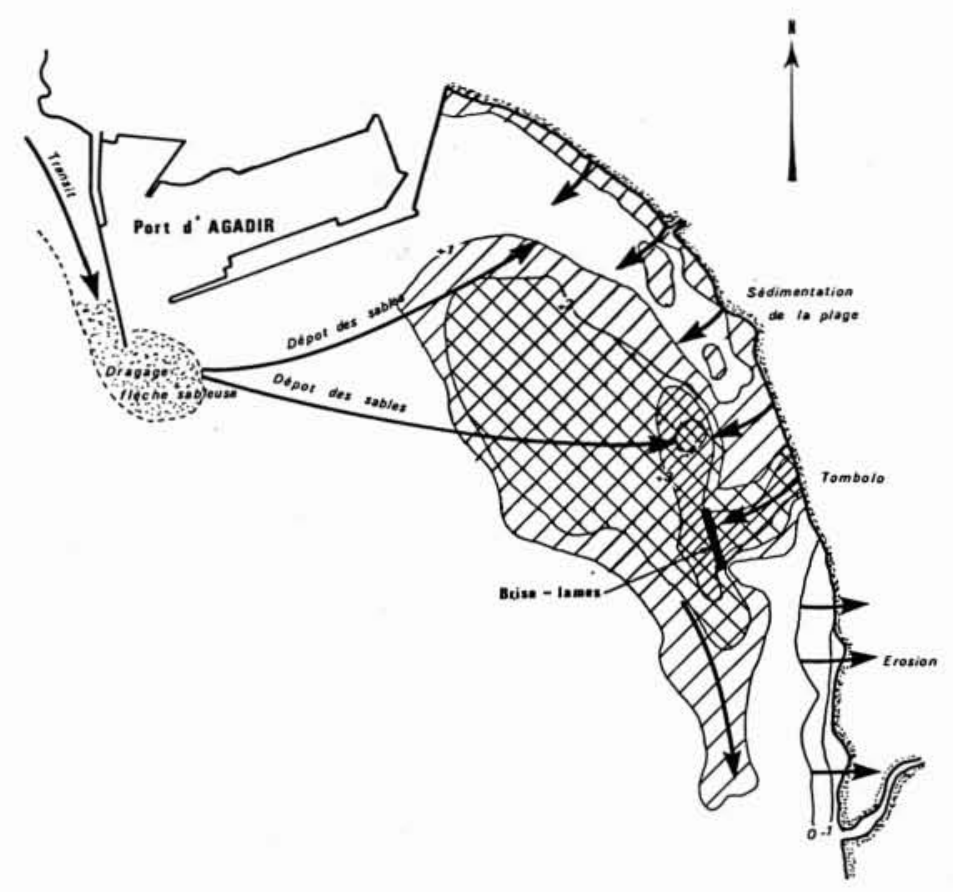

34. Engraissement des fonds de la plage d'Abidjan entre 1962 et 1972

\subsection{Impact sédimentologique des ouvrages portuaires arrê- tant en grande partie le transit littoral}

La réalisation et les extensions des ouvrages portuaires de Safi, Agadir et Laayoune ont apporté des perturbations notables dans les mouvements de sable comme sur le régime de l'agitation sur le littoral avoisinant les ports, côté Sud. En effet l'ensemble de ces aménagements accumulait le transit littoral pratiquement en totalité dans la zone Nord. Sur les zones Sud sous alimentées et soumises à une agitation renforcée par l'érosion et la présence des ouvrages, le littoral a connu un recul important (sur 5 à $7 \mathrm{~km}$ ) pouvant atteindre juqu'à $10 \mathrm{~m} / \mathrm{an}$ à Agadir sur les zones sollicitées et varie en général entre 2 et $5 \mathrm{~m} / \mathrm{an}$.

Sur les plages d'Agadir et Laayoune, l'érosion est accentuée par les transports éoliens.

A Safi, les solutions consistant à rejeter les produits de dragages ( $\left.250000 \mathrm{~m}^{3} / \mathrm{an}\right)$ en face du Château portugais par profondeurs moyennes n'ont pas donné une efficacité satisfaisante, puisque la zone de rejet se situait au large de la zone de déferlement.

A Agadir le tronçon de plage s'étendant du port ancien à la station de pompage a été considérablement réengraissé après la réalisation d'un brise-lames de $200 \mathrm{~m}$ de longueur par des fonds de $-3,50 \mathrm{~m}$ à $-4 \mathrm{~m}$ et en déversant des produits de dragages ( 350000 à $400000 \mathrm{~m}^{3} / \mathrm{an}$ ) par petits fonds $(-2 \dot{a}-2,5 \mathrm{~m})$ au Nord du brise-lames (fig. $32 \dot{a}$ 34).
Ces dispositions n'ont eu aucun effet sur le tronçon de plage au Sud du brise-lames qui a continué son érosion, au taux de 2,5 m/an. Par contre, l'arrêt des rechargements au Nord du brise-lames, l'action éolienne et la réalisation du nouveau port d'Agadir en (1984-1986) ont accentué les phénomènes d'érosion au Sud du brise-lames et le développement de la zone érodée a connu des extensions jusqu'à l'Oued Souss. La zone au Nord du brise-lames se trouvant à l'abri du complexe portuaire est restée stable malgré l'arrêt des rechargements.

Les solutions de protection doivent systématiquement prendre en considération le rétablissement du transit littoral de la zone amont vers la zone aval des ouvrages soit par dragages, pompages à postes fixes ou by-pass (avec rejets par petits fonds à terre de la zone de déferlement soit sur l'estran en plusieurs endroits).

Les études sur modèle réduit sont nécessaires pour préciser les solutions à adopter pour le rétablissement du transit, et définir si nécessaire la mise en place des ouvrages complémentaires longitudinaux ou transversaux pour maintenir l'équilibre sédimentaire du littoral. Les prévisions faites par de telles études sur modèles réduits physiques sédimentologiques se sont trouvées dans presque tous les cas confirmées par les évolutions des fonds constatées en nature après réalisation des ouvrages portuaires maritimes ou de défense du littoral le long des côtes marocaines. 